\title{
Energy-Efficient Relay-Based Void Hole Prevention and Repair in Clustered Multi-AUV Underwater Wireless Sensor Network
}

\author{
Amir Chaaf, ${ }^{1}$ Mohammed Saleh Ali Muthanna ${ }^{1},{ }^{2}$ Ammar Muthanna, ${ }^{3,4}$ Soha Alhelaly, ${ }^{5}$ \\ Ibrahim A. Elgendy $\mathbb{D}^{6},{ }^{6}$ Abdullah M. Iliyasu $\mathbb{D},,^{7,8,9}$ and Ahmed A. Abd El-Latif $\mathbb{C}^{10}$ \\ ${ }^{1}$ School of Communication and Information Engineering, Chongqing University of Posts and Telecommunications, \\ Chongqing, China \\ ${ }^{2}$ School of Computer Science and Technology, Chongqing University of Posts and Telecommunications, Chongqing, China \\ ${ }^{3}$ Department of Telecommunication Networks and Data Transmission, \\ The Bonch-Bruevich Saint-Petersburg State University of Telecommunications, 193232 Saint Petersburg, Russia \\ ${ }^{4}$ Department of Applied Probability and Informatics, Peoples' Friendship University of Russia (RUDN University), \\ 6 Miklukho-Maklaya St, Moscow 117198, Russia \\ ${ }^{5}$ College of Computing and Informatics, Saudi Electronic University, Riyadh, Saudi Arabia \\ ${ }^{6}$ School of Computer Science and Technology, Harbin Institute of Technology, Harbin, China \\ ${ }^{7}$ Electrical Engineering Department, Prince Sattam Bin Abdulaziz University, Al-Kharj 11942, Saudi Arabia \\ ${ }^{8}$ School of Computing, Tokyo Institute of Technology, Yokohama 226-8502, Japan \\ ${ }^{9}$ School of Computer Science and Technology, Changchun University of Science and Technology, Changchun 130022, China \\ ${ }^{10}$ Mathematics and Computer Science Department, Faculty of Science, Menoufia University, Shebin El-Koom, Egypt
}

Correspondence should be addressed to Ahmed A. Abd El-Latif; a.rahiem@gmail.com

Received 16 March 2021; Accepted 17 June 2021; Published 1 July 2021

Academic Editor: Leo Y. Zhang

Copyright (C) 2021 Amir Chaaf et al. This is an open access article distributed under the Creative Commons Attribution License, which permits unrestricted use, distribution, and reproduction in any medium, provided the original work is properly cited.

Underwater wireless sensor networks (UWSNs) enable various oceanic applications which require effective packet transmission. In this case, sparse node distribution, imbalance in terms of overall energy consumption between the different sensor nodes, dynamic network topology, and inappropriate selection of relay nodes cause void holes. Addressing this problem, we present a relay-based void hole prevention and repair (ReVOHPR) protocol by multiple autonomous underwater vehicles (AUVs) for UWSN. ReVOHPR is a global solution that implements different phases of operations that act mutually in order to efficiently reduce and identify void holes and trap relay nodes to avoid it. ReVOHPR adopts the following operations as ocean depth (levels)based equal cluster formation, dynamic sleep scheduling, virtual graph-based routing, and relay-assisted void hole repair. For energy-efficient cluster forming, entropy-based eligibility ranking (E2R) is presented, which elects stable cluster heads (CHs). Then, dynamic sleep scheduling is implemented by the dynamic kernel Kalman filter (DK2F) algorithm in which sleep and active modes are based on the node's current status. Intercluster routing is performed by maximum matching nodes that are selected by dual criteria, and also the data are transmitted to AUV. Finally, void holes are detected and repaired by the bicriteria mayfly optimization (BiCMO) algorithm. The BiCMO focuses on reducing the number of holes and data packet loss and maximizes the quality of service (QoS) and energy efficiency of the network. This protocol is timely dealing with node failures in packet transmission via multihop routing. Simulation is implemented by the NS3 (AquaSim module) simulator that evaluates the performance in the network according to the following metrics: average energy consumption, delay, packet delivery rate, and throughput. The simulation results of the proposed REVOHPR protocol comparing to the previous protocols allowed to conclude that the REVOHPR has considerable advantages. Due to the development of a new protocol with a set of phases for data transmission, energy consumption minimization, and void hole avoidance and mitigation in UWSN, the number of active nodes rate increases with the improvement in overall QoS. 


\section{Introduction}

Underwater wireless sensor network (UWSN) has many applications over the ocean environment. In UWSN, energy efficiency is the major constraint since the nodes are resource constraint [1-3]. This represents one of the main reasons that leads to the appearance of void holes, reducing the performance of the network. To achieve energy efficiency, various approaches were presented in UWSN. Here, the data transmission is carried over multiple hops between a number of sensor nodes through a selected route to reach the autonomous unmanned vehicles (AUVs), and then the final surface sink node and further collision-free medium access (MAC) protocols were presented. However, routing is also the best way to improve energy efficiency [4]. A clusterbased mobile data gathering is used to improve energy efficiency in the large-scale network [5]. The basic cluster concept is considered in this work to form initial clusters $[6,7]$. This cluster formation is performed in nonoptima manner which is inefficient [8]. However, cluster head $(\mathrm{CH})$ is performed in a random manner which makes this work ineffectual [9]. In addition, processing the distributed clustering algorithm needs a large amount of control packet exchange which consumes lots of energy. Autonomous unmanned vehicles (AUVs) are specially designed for data gathering in the underwater environment [10-12]. An AUVassisted energy-efficient clustering UWSN mechanism faces many serious issues as follows $[13,14]$ :

(i) Energy consumption in existing research works is high, which leads to a large number of holes in the network.

(ii) Network clusters with unequal size introduce energy imbalance in certain regions, leads to a large number of holes.

(iii) Optimal sleep scheduling is necessary in order to reduce the energy consumption of the nodes and avoid holes.

(iv) Route selection considers only limited metrics, which leads to large packet loss and energy consumption which induces trap nodes.

In AUV-assisted UWSN, the predefined path determination is the critical issue which increases the distance to the nodes, the energy consumption, and delay in data transmission $[15,16]$. On the other hand, the unnecessary sensing of the sensor nodes increases energy consumption. These are only limited factors since the forwarder selection mechanism must consider more criteria. Furthermore, route selection based on single metric is ineffective in underwater scenarios $[17,18]$. Traditional routing algorithms follow ocean depthbased routing. This leads to high packet loss due to the void hole issue. Void hole avoidance and recovery is an emerging part of UWSN. Furthermore, it occurs frequently in the sparse node distribution with a limited amount of energy. In addition, various important issues remain untouched in UWSN for reducing energy consumption and avoiding energy hole creation $[19,20]$. Table 1 describes the abbreviations that we have used throughout the paper:
TABLE 1: List of abbreviations.

\begin{tabular}{|c|c|}
\hline Abbreviation & Expansion \\
\hline UWSN & Underwater wireless sensor network \\
\hline AUVs & Autonomous unmanned vehicles \\
\hline ReVOHPR & $\begin{array}{l}\text { Relay-based void hole prevention and repair } \\
\text { protocol }\end{array}$ \\
\hline DK2F & Dynamic kernel Kalman filter \\
\hline $\mathrm{BiCMO}$ & Bicriteria mayfly optimization \\
\hline EEDG & Energy-efficient data gathering \\
\hline $\mathrm{E} 2 \mathrm{R}$ & Eligibility ranking \\
\hline $\mathrm{CH}$ & Cluster head \\
\hline LECA & Level-based equal clustering algorithm \\
\hline AEC & Energy-efficient clustering \\
\hline MFO & Moth flame optimization \\
\hline CMDG & Cluster-based mobile data gathering \\
\hline
\end{tabular}

(i) There is no unified protocol for reliable and energyefficient data transmission for a specific type of UWSN.

(ii) Existing protocols focus on one aspect for energy consumption, i.e., clustering, routing, or void hole repair. Hence, energy consumption may occur by other aspects of the issue.

(iii) Current protocols used a single AUV for data collection, which increases the end-to-end delay of each sensor, and thus, energy consumption rate is increased $[21,22]$.

1.1. Motivation. Figure 1 illustrates the void hole problem in UWSN. In UWSN, the presence of routing void holes leads to higher packet loss which makes the data unreliable. The main cause for routing voids is the higher energy consumption of the sensor nodes in the network, i.e., nodes which lose energy makes the hole. In this context, there are two major research problems arise [23-25]:

(i) Most of the works have concentrated on energyefficient route selection without deploying AUV in the network. In this case, the energy consumption and delay for data transmission is high. Although these works select optimal route, it fails to transmit the data in a timely manner since the hole mitigation process generally transmits the data in longest path or backward path.

(ii) In some works, AUVs are deployed to mitigate the problem of void holes. However, there is an issue in predicting the trajectory of the AUVs since the travel length is high. As the trajectory detection methods use the energy level alone for optimal positioning to collect the sensor's data.

(iii) The network is managed with unequal clusters which imbalances the load among clusters. Thus, some of the cluster heads suffer form higher energy consumption while some cluster heads suffer from lower energy consumption. In general, all underwater sensor nodes are continuously sensing the 


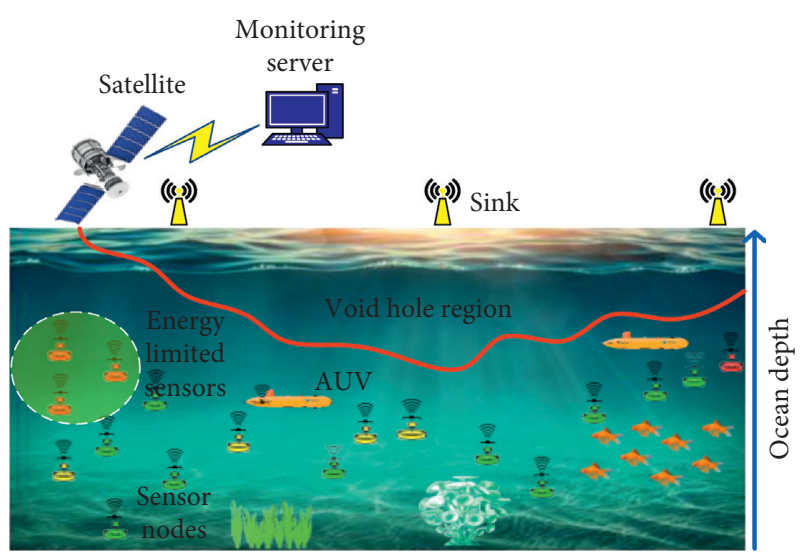

FIgURE 1: Void hole problem in UWSN.

environment, which consumes a lot of energy and introduces void holes. In the absence of an optimal sleep scheduling mechanism, the energy consumption will be high and the holes are unavoidable.

This paper mainly focuses on void hole prevention and mitigation in underwater wireless sensor networks (UWSNs). For that, we intend to combine intercluster routing performance and a relay-assisted void mitigation mechanism initiated by an AUV according to an optimal trajectory and a positioning of a suitable relay node for data transmission continuity. We also prevent the holes by minimizing overall energy consumption in the network by introducing multiple mechanism clustering, sleep scheduling. This research work is motivated from the problems presented in the existing research works.

1.2. Contributions. In this paper, we deeply tackle all the issues of reducing the energy consumption in UWSN. In particular, we presented the following contributions to address the energy consumption and QoS issues:

(i) The level-based equal clustering algorithm (LECA) is presented that utilizes distance and load criteria. In each level, equal clusters are formed to avoid energy consumption. Entropy-based eligibility ranking (E2R) protocol is presented for $\mathrm{CH}$ selection. Optimal $\mathrm{CH}$ is selected in two levels based on energy, centrality, and success rate which increase the lifetime of $\mathrm{CH}$.

(ii) To reduce energy consumption, dynamic sleep scheduling is presented by the dynamic kernelbased Kalman filter (DK2F) which is proposed. This considers residual energy, buffer value, and coverage rate to make the decision on node status (sleep/ active).

(iii) Optimal route is selected in multiple hops by considering multiple factors. A virtual graph-based routing is presented, which uses maximum matching theory for optimum selection of next hops. Optimal positioning of relay nodes by the
AUV repairs the voids in the network. Proposed maximum matching approach chooses optimal criteria according to the position of AUV.

(iv) The optimal repair position initiated by the AUV by repositioning relay nodes is computed by the mayfly optimization algorithm which is proposed and works upon multiple criteria to find the optimal trajectory of AUV and the repositioning of relay nodes. The mayfly algorithm works well in terms of convergence rate and convergence time.

(v) Overall network follows level-based clustering and optimal routing, which minimizes the energy consumption that prevents the holes. Sleep scheduling prevents the nodes from dead, which further prevents the hole.

1.3. Paper Layout. The remaining part of the paper is organized as follows: Section 2 presents the literature review in the area of clustering, routing, sleep scheduling, and void hole detection and repair in UWSN. Section 3 focuses on the problems that existed previously in void hole avoidance. Section 4 describes the research methodology, pseudocode, and algorithms in details. Section 5 illustrates the performance of network simulation for the proposed and previous protocols. Section 6 concludes the paper and presented the future works.

\section{Lietarture Review}

An energy-efficient data gathering (EEDG) scheme was proposed in [26] for the underwater wireless sensor network. The data transmission is performed in a multihop manner. At first, the energy consumption is balanced by grouping the nodes into smaller groups. Furthermore, the forwarder nodes are selected to gather the data from the subset nodes. Here, the communication is carried out in a one-hop manner. Furthermore, a medium access control (MAC) protocol is utilized to improve collision rate and packet loss.

A fault resilient routing for the underwater wireless sensor network was presented in [27] for underwater data transmission. The fault-tolerant routing follows the moth flame optimization (MFO) algorithm. The data transmission is carried through AUVs to base stations. Here, the AUVs act as cluster heads that are responsible to collect data from the sensor nodes. The use of AUVs avoids reclustering and overloading problems. To overcome the path disjoint issues, additional mobile nodes are deployed in the network. In this work, multiple AUVs are deployed to support data forwarding in the underwater network.

A cluster-based mobile data gathering (CMDG) scheme was studied in [28] for the large-scale underwater sensor network. At first, the cluster formation and $\mathrm{CH}$ selection problem is formulated as an optimization problem. In this work, the AUV tour planning scheme is presented to handle the sensor mobility. In order to achieve an energy-latency tradeoff, the travel length is shortened for AUV movement. A centralized clustering algorithm is proposed to form initial clusters. Then, the distributed clustering algorithm is 
proposed to maintain the formed clusters. This work has two drawbacks as follows: (1) $\mathrm{CH}$ selection is inefficient since it considers only a minimum number of parameters and (2) the distributed clustering algorithm exchanges a large number of control packets, which is ineffective, and the consumption of higher energy and delay on sensor nodes due to data transmission.

AUV-assisted energy-efficient clustering (AEC) mechanism was presented in [29]. The proposed AEC mechanism introduces wake-up sleep cycle for the underwater sensor network. The overall mechanism includes cluster formation, cluster head nomination, and sleep wake-up scheduling. To form clusters, virtual sectoring approach is presented. In each virtual sector, the cluster is formed and a $\mathrm{CH}$ is selected. The $\mathrm{CH}$ is selected based on the distance with the cluster centroid point. Then, the path of AUV is a predefined path. The predefined path of AUV is inefficient since it consumes large amount of energy and increases delay. The $\mathrm{CH}$ selection was poor due to the estimation of only energy.

Author proposes a cluster-based sleep scheduling mechanism in UWSN [30]. The overall network is considered as the 3D underwater sensor network. A 3D partition unit is considered with a basic cluster structure. All sensor nodes are in the temporary control of clusters. In each cluster, sleep-awake scheduling is enabled based on the remaining energy level. The major goal of this work is to achieve minimum energy consumption and guarantee maximum sensing coverage in the network. Cluster formation in performed in a nonoptimal manner which is inefficient.

In [31], the authors propose a two-stage routing protocol. The main purpose of this protocol is to enable communication between not only connected nodes but also for nonconnected or partially connected nodes so that the packet delivery rate will be improved. To delay the death of nodes, an energy threshold method and rerouting scheme is proposed. Involvement of energy threshold and rerouting processes improve the connectivity of the network, which prevents the holes, and preserve resource constraints, forwarding loops. However, unnecessary sensing of the sensor nodes increases the energy consumption.

An energy-balanced efficient and reliable routing (EBER2) protocol for UWSNs was presented in [32]. Energy balancing among neighbors and reliability are achieved in EBERR protocol. The EBERR protocol considers residual energy and potential forwarding nodes (PFNs) of the forwarder node. The transmission range is divided into power levels, and the forwarders can adjust the transmission range adaptively. In order to suppress the duplicate packets, depth of the nodes is compared. Forwarder selection is inefficient since it considers only limited metrics, and also the sensor nodes nearer to the sink will drain with larger energy due to the transmission of sensed data from the nodes in the network.

A distance vector-based opportunistic routing (DVOR) protocol was proposed to address the problem of void regions in the underwater sensor network [33]. The main idea behind this protocol is to use the depth information for route selection. The DVOR uses a query-based mechanism to enable the distance vectors for underwater sensor networks.
From the distance vectors, each node records the smallest hop count information towards sink node. Based on this hop count information, routing is performed. The void hole is avoided by selecting a route with small distance. When the network is sparse, then the hop count information will have a large distance which increases the energy consumption. Optimal route selection based on single metric is insufficient to cope with UWSN.

In [34], sink mobility, i.e., AUV, and courier nodes are deployed in network for data collection, aggregation, and transmission. The entire network is divided into four sectors. Both courier nodes and AUV are movable with random trajectory. In this protocol, routing is fixed and sink mobility is dynamic. Comparison is made between several existing protocols and the proposed protocol. However, the mobility of mobile sink and courier nodes increases energy consumption and decrease the network lifetime. This is a linear type of network, it does not suit for complex ocean depth scenarios, and also realistic applications are not adopted with this protocol. In [35], the authors proposed a new data collection protocol for QoS provisioning. For that, the bioinspired routing algorithm is proposed which facilitates the natural features of the genetic algorithm. Clusters are formed which provides a highly stable and different size of clusters for traffic load balancing. The proposed routing algorithm predicts high stable links as a forwarding node. This work eliminates the data transmission by the upward and downward transmission. The main drawback of this protocol is that it is not aware of node mobility and packet delivery. In [36], fuzzy clustering is presented, which designs the fitness function for selecting the $\mathrm{CH}$ according to the distance between the nodes. For cluster formation, the fuzzy algorithm is used, whereas CHs are selected by the PSO algorithm. The overall network topology is arranged in a hierarchical structure, and the comparative analysis is made between the proposed hybrid (fuzzy and PSO) algorithm with LEACH and traditional PSO algorithms. This hybrid protocol has several drawbacks:

(i) Hybrid fuzzy and PSO algorithms consume more energy by underwater sensors since the computation of both algorithms is very high. Due to limited battery issue of underwater sensors, this protocol is not suited.

(ii) The overall work partially reduces the energy level, which does not suit risky oceanic applications, and also, it does not increase the lifespan of the UWSN.

In [37], the void hole alleviation issue is addressed using enhanced geographic and opportunistic routing protocol in the harsh underwater WSN. There are three problems, such as void hole occurrence, higher energy consumption, and low packet delivery rate that are addressed in this paper. Furthermore, the network scalability issue is addressed in this paper. The performance of the proposed protocol is compared with the geographic and opportunistic routing with topology control protocol based on depth adjustment as well as transmission-adjusted neighbor node approaching distinct algorithms with energy-efficient mate. 
Table 2 shows a comparison summary of various existing works [28-33] that have studied the main elements of energy consumption minimization and void hole avoidance and mitigation in UWSN that address the critical issues related to clustering, node sleep scheduling, routing, and hole mitigation.

\section{Problem Statement}

The purpose of this paper is to investigate the problem of void hole and repair in multi-AUV-enabled UWSN. This section summarizes the important issues in current works.

Authors in [38] focus on void hole detection and mitigation in an underwater sensor network. For that, two routing schemes are proposed. The first routing scheme, called energy-aware scalable reliable and void hole mitigation routing (ESRVR), intends to avoid holes during route selection. In that, the two-hop neighbor information is collected before initiation of route selection. As this scheme considers two hops, the void hole is avoided in the route selection itself. The second scheme, namely, cooperative ESRVR (Co-ESRVR), focuses on mitigating void holes through backward transmission. The major drawbacks of this paper are follows:

(i) Two-hop neighbor information alone is insufficient to avoid holes since the data from the nodes that are deployed in deep level need to transmit through multiple hops. Thus, there is a need for gathering multihop information which is not efficient.

(ii) In the sparse network environment, it is hard to gather two-hop neighbor information, since it is not sure that always two-hop nodes will be presented.

(iii) In backward routing energy consumption and delay is high. That is Co-ESRVR scheme also introduces multiple holes that need to be mitigated.

(iv) When the network is sparse, or there is a limited possibility for backward transmission, then the ESRVR and Co-ESRVR are not feasible.

In [39], the authors propose a game-theoretic approach for energy-efficient routing in the 3D underwater WSN. However, game theory approaches have several drawbacks, which are as follows:

(i) The game played by the nodes is noncooperative. Thus, the strategy played by the players is unknown to other nodes. It leads to the same route is selected by multiple source nodes, which introduces a large number of collisions. Due to collisions, packet retransmission count is high. This leads to large energy consumption.

(ii) The game theory approach has high complexity. In addition, all players in the forwarding region are considered (other than neighbors) which makes the algorithm more complex.

Hence, high complexity in game theory approach requires more energy by sensors and also takes higher processing time. In [40], the authors propose an AUV-assisted data gathering approach to minimize energy consumption in Smart Ocean. For that, an AUV-assisted data gathering scheme based on the clustering and matrix completion (ACMC) method for UWSN is proposed. The drawbacks in this work are the follows:

(i) The K-means algorithm forms clusters based on distance value. The formed unequal clusters will have imbalanced load among clusters. Presence of an imbalanced load leads to energy consumption in some regions.

(ii) $\mathrm{CH}$ and secondary $\mathrm{CH}$ are selected in each cluster. Here, the cluster center criteria are considered for $\mathrm{CH}$ selection while secondary $\mathrm{CH}$ is selected in a random manner. Thus, $\mathrm{CH}$ selection is inefficient as the selected $\mathrm{CH}$ may lose the energy, which becomes void holes.

(iii) The greedy algorithm-based AUV trajectory only considers the trajectory length. This means that nearby position is selected as AUV moving position. It does not make decisions based on the energy level of the nodes. If the nodes with low energy are located far away from the current AUV position, then there will be higher energy consumption. The greedy algorithm has higher time consumption and complexity.

In [41], the authors proposed an AUV-assisted void prediction and repair mechanism in the underwater sensor network. The repair position is calculated by the particle swarm optimization (PSO) algorithm. In this paper, PSObased void prediction causes more issues that are listed as follows:

(i) Repair position for AUV is computed by PSO, which traps the solutions into local optima. It leads to the nonoptimal positioning of AUV.

(ii) Here, the single AUV collects the repair requests from multiple sensor nodes. And the rules are predefined. When the number of holes in the network is large, then the AUV suffers in decisionmaking. The AUV could not handle a large amount of requests from the sensor nodes. Thus, this work is unable to mitigate the void holes effectively.

(iii) This work only mitigates the void but unable to prevent the voids due to a lack of optimal clustering and route selection procedures. The main reason for holes is high energy consumption. In this work, energy consumption is high in data collection, data transmission, and sensing.

Based on the shortcomings and issues cited, we aimed to design a global solution by introducing an efficient clustering method on the sensor nodes, an optimal cluster head selection, and also an efficient sleep scheduling method to avoid the continuous sensing of the sensors to reduce the overall energy consumption which also decreases the creation of void holes accordingly, a routing method is also used to gather and transmit the sensors data between the clusters 
TABLE 2: Summarize the contribution of existing works and comparison between them.

\begin{tabular}{|c|c|c|c|c|c|}
\hline $\begin{array}{l}\text { Existing } \\
\text { work }\end{array}$ & Clustering & $\begin{array}{l}\text { Node sleep } \\
\text { scheduling }\end{array}$ & Routing & $\begin{array}{c}\text { Hole } \\
\text { mitigation }\end{array}$ & Key contributions and limitations \\
\hline [28] & $\checkmark$ & $\checkmark$ & $x$ & $x$ & $\begin{array}{l}\text { (i) Propose a clustering scheme and a } \mathrm{CH} \text { selection to gather the sensor } \\
\text { data from each cluster and deploy an AUV with a tour planning } \\
\text { scheme to collect data from CHs. } \\
\text { (ii) Finding a tradeoff between consumption of energy and data } \\
\text { gathering delay. } \\
\text { Limitations: CH selection is inefficient since it considers only a } \\
\text { minimum number of parameters, and distributed algorithm exchanges } \\
\text { large number of control packets which is ineffective. }\end{array}$ \\
\hline
\end{tabular}

(i) Introduces a wake-sleep cycle for the sensors to reduce their energy consumption.

[29]

$\checkmark \quad \checkmark$

$\checkmark \times$

$x+x$

(ii) A virtual sectoring approach is presented, cluster is formed, and $\mathrm{CH}$ is selected to reduce energy consumption; the $\mathrm{CH}$ data will be gathered by a predefined path of the AUV.

Limitations: the predefined path of AUV is inefficient since it consumes large amount of energy by the $\mathrm{CH}$ s to transmit their data and increases the delay.

(i) The major goal of this work is to achieve minimum consumption of energy and guarantee maximum sensing coverage in the network. Limitations: cluster formation is performed in a nonoptimal manner which is inefficient.

\begin{tabular}{lllll} 
[30] & $\checkmark$ & $\checkmark$ & $\times$ & $\times$ \\
\hline
\end{tabular}

(i) The main goal of this protocol is to enable communications between not only connected nodes but also for nonconnected or partially connected nodes so that the packet delivery rate will be improved.

[31] $\times$

$\times$

$\checkmark$

(ii) To delay the death of nodes, an energy threshold method and a rerouting scheme are proposed.

Limitations: unnecessary sensing of the sensor nodes increases the energy consumption.

(i) The main goal is to achieve the energy balancing among neighbor nodes and reliability.

\section{[32]}

$\times$

$\times$

$\checkmark$

$\times$

[33]

$x+10 x+10$
Limitations: during the data transmission process, forwarder selection is inefficient since it considers only limited metrics.

(i) The main ideas behind this protocol are to use the depth information for route selection.

(ii) The void hole is avoided by selecting a route with small distance. Limitations: when the route is sparse, then the hop count information will have a large distance, which increases the energy consumption. Optimal route selection based on a single metric is insufficient to cope with underwater sensor network.

efficiently, and introducing the use of multi-AUV aims to detect and repair the creation of the hole according to the current trajectory position of the AUV to mobile relays that are used as a replacing part of the failed node and an intermediate receiver of the data from the cluster heads to the AUV, which will be selected and repositioned by the AUV. By combining all the presented solutions, the decrease of the overall energy consumption, reducing, detecting, and repairing of the void holes will be ensured.

\section{System Model}

4.1. System Overview. In this work, we present an energyefficient relay-assisted 3D-UWSN model that absorbs the surrounding by collecting data and transmitting the information in which void hole prevention and mitigation procedure is focused. The overall 3D-UWSN is constructed as $x_{i}, y_{i}, z_{i}$ coordinates, and this $3 \mathrm{D}$ network is divided into multiple levels as $L_{1}, L_{2}, \ldots, L_{\mathrm{N}}$ based on the depth of the ocean covering shallow water and deepwater areas. Each level LN is composed of $n$ number of sensors $L_{N}=\left(n_{1}, n_{2}, \ldots\right.$, $\left.n_{N}\right)$. The network model comprises underwater sensor nodes, sink node, mobile relays, and multiple AUVs. We construct the network based on multiple levels. In each level, an AUV is deployed to gather data from the underwater sensor nodes, and AUVN collects data from $L_{N}$ level. All the nodes have the same initial energy $E_{\text {Ini }}$ and the sensing range $R_{\mathrm{S}}$. Sensors can be transmitted into two types as topology information and event. The size of the event and topology information packages is $M$. The network connectivity rate is defined as the ratio of $S_{C}$ which is computed by $N(S)_{C}$ (number of sensors that communicates with the sink node) via single hop, which means that the sensors can achieve their sensed data directly to the surface sink that is present in their coverage range and a multihop communication which means that the sensors transmit their collected data through 
other sensor nodes by constituting a route to achieve the data to the final sink and it is computed as follows:

$$
S_{C}=\frac{N(S)_{C}}{n} \text {. }
$$

When the network connectivity is 1 , the network can obtain the full network connectivity, and all sensors can communicate with the surface sink with either one-hop or multihop communication.

The overall process comprises four major phases that are explained in the following sections. Figure 2 describes the overall network model.

4.2. Level-Based Equal Cluster Formation. The overall network is segregated into multiple equal clusters based on the depth of the underwater environment. We propose a new level-based equal clustering algorithm (LECA). Generally, the nodes presented in underwater-based sensor networks are considered resource-constrained (i.e., battery-powered nodes). The node cannot participate in the network if the level of energy for that node is drained. Therefore, the selected node should have sufficient energy for transmitting the data. Thus, E2R considers the node's residual energy as another metric. Thereby, the sensor with the more residual energy has the large possibility to be $\mathrm{CH}$.

4.2.1. Energy Consumption Model. Significant efforts have been made to address the UWSN's energy consumption, in which all nodes in the network are energy constraint (i.e., the energy sources of the nodes drop by usage). In addition, the large consumption of energy leads to early dead which decreases the lifetime of the network. The consumption of energy for the node $n_{i}$ can be calculated as

$$
E\left(n_{i}\right)=E_{\text {idle }}\left(n_{i}\right)+\sum_{v \in V} \sum_{p \in P(v)} w(p) \times A(v) \times E\left(n_{i}, p\right) .
$$

Assuming the path $p \in P(v)$, then the path weight $w(p)$ is expressed as

$$
\sum_{p \in P(v)} w(p)=1
$$

where $A(v)$ denotes the average amount of consumed energy through the node $n_{i}$ for a time unit regarding the transmission of data, $E\left(n_{i}, p\right)$ denotes the amount of consumed energy only either in reception pr transmission, and $E_{\text {idle }}$ denotes the average amount of consumed energy at the idle state by the node $n_{i}$ per unit time. Regarding the estimated consumption energy for the node, the node's lifetime is expressed as

$$
\operatorname{LT}\left(n_{i}\right)=\frac{E_{\text {ini }}}{E\left(n_{i}\right)}
$$

where $E_{\text {ini }}$ denotes the sensor node's initial energy, which is initially fed into the node for its network's participation. The proposed LECA divides the network environment into multiple levels as $L_{1}, L_{2}, \ldots, L_{n}$. In each level, clusters are formed based on the load level. Then, each cluster performs entropy-based eligibility ranking (E2R)-based $\mathrm{CH}$ selection protocol to select optimal $\mathrm{CH}$. Here, Tsallis entropy is utilized to formulate an optimal energy threshold. Upon threshold value, candidate nodes are filtered by E2R protocol. The proposed E2R protocol uses residual energy level $\boldsymbol{R}_{\xi}$, centrality factor $\mathbf{C}_{\mathbf{f}}$, and success rate criteria $\mathbf{S R}_{\mathbf{c}}$.

Algorithm 1 explains the procedure of E2R algorithmbased cluster formation and $\mathrm{CH}$ selection. Involvement of the E2R algorithm improves the data aggregation process as well as network QoS performance. Furthermore, involvement of E2R-based data aggregation also helps to minimize the risk of instability in the network. Generalized Tsallis entropy is computed by

$$
T_{Q}\left(P_{1} \ldots P_{W}\right)=\frac{1}{1-q}\left(\sum_{i=1}^{w} p_{i}^{q}-1\right),
$$

where $q$ is the logarithmic function. For all sensors in the network, $T_{Q}\left(P_{1} \ldots P_{W}\right)$ is computed for the number of given input parameters. Using $T_{Q}$, the weight value for $\mathrm{CH}$ selection is implemented as

$$
T_{Q}=\left(S_{1} \cdot C_{1}\right) w_{1} \times\left(S_{2} \cdot C_{2}\right) w_{2} \times\left(S_{3} \cdot C_{3}\right) w_{3},
$$

where $w_{1}, w_{2}$, and $w_{3}$ are the weight values for $C_{1}, C_{2}$, and $C_{3}$, respectively.

The $\mathrm{CH}$ is selected based on

$$
\mathrm{CH}_{\text {prob }}=\max \left(C_{f \text { prob }} \mathfrak{R}_{\xi \max }, \mathrm{SR}_{c \max }\right) .
$$

After $\mathrm{CH}$ selection, clusters are formed based on sensor node's cost function as

$$
\mathrm{CM}(i, j)=\frac{\mathrm{SR}_{c(i j)}}{\mathrm{SR}_{c(\max )}} * \frac{E_{\mathrm{cur}}(i)}{E_{\mathrm{cur}}(j)} * \frac{C(i j)}{C_{\mathrm{ave}}},
$$

where $E_{\text {cur }}(i)$ represents the current energy of node $i$ and $E_{\text {cur }}(j)$ represent the current energy of $\mathrm{CH} j$. Each $\mathrm{CH}$ in the cluster aggregates the sensor data and forwards them to the AUV. The proposed E2R protocol has $O(n)$ complexity which is due to the message transmission overhead where $n$ is the number of sensors. $\mathrm{CH}$ announces, join request, and join response messages are exchanged within the cluster, which introduces the complexity in cluster.

4.3. Dynamic Sleep Scheduling. In each cluster, a dynamic sleep scheduling procedure is established in order to reduce the energy consumption of the sensor nodes by avoiding their continuous sensing of the environment which causes an unnecessary energy consumption that leads to void holes. By taking into consideration different factors on the nodes, the decision on the nodes status (sleep, awake, and idle) can be taken effectively. For that, we propose the dynamic kernel Kalman filter (DK2F) algorithm. In particular, Cauchy kernel function is used for nonlinear cases. The DK2F algorithm takes residual energy level $\mathfrak{R}_{\xi}$, buffer factor $\varsigma_{f}$, and coverage rate $\chi_{r}$ to make decision on the node status. The considered statuses are sleep, active, and transmit. The DK2F is executed by each node, and the report is sent to the $\mathrm{CH}$. Then, the $\mathrm{CH}$ activates the mode for each node in the cluster. The procedure for $\mathrm{DK} 2 \mathrm{~F}$ is described as follows. 


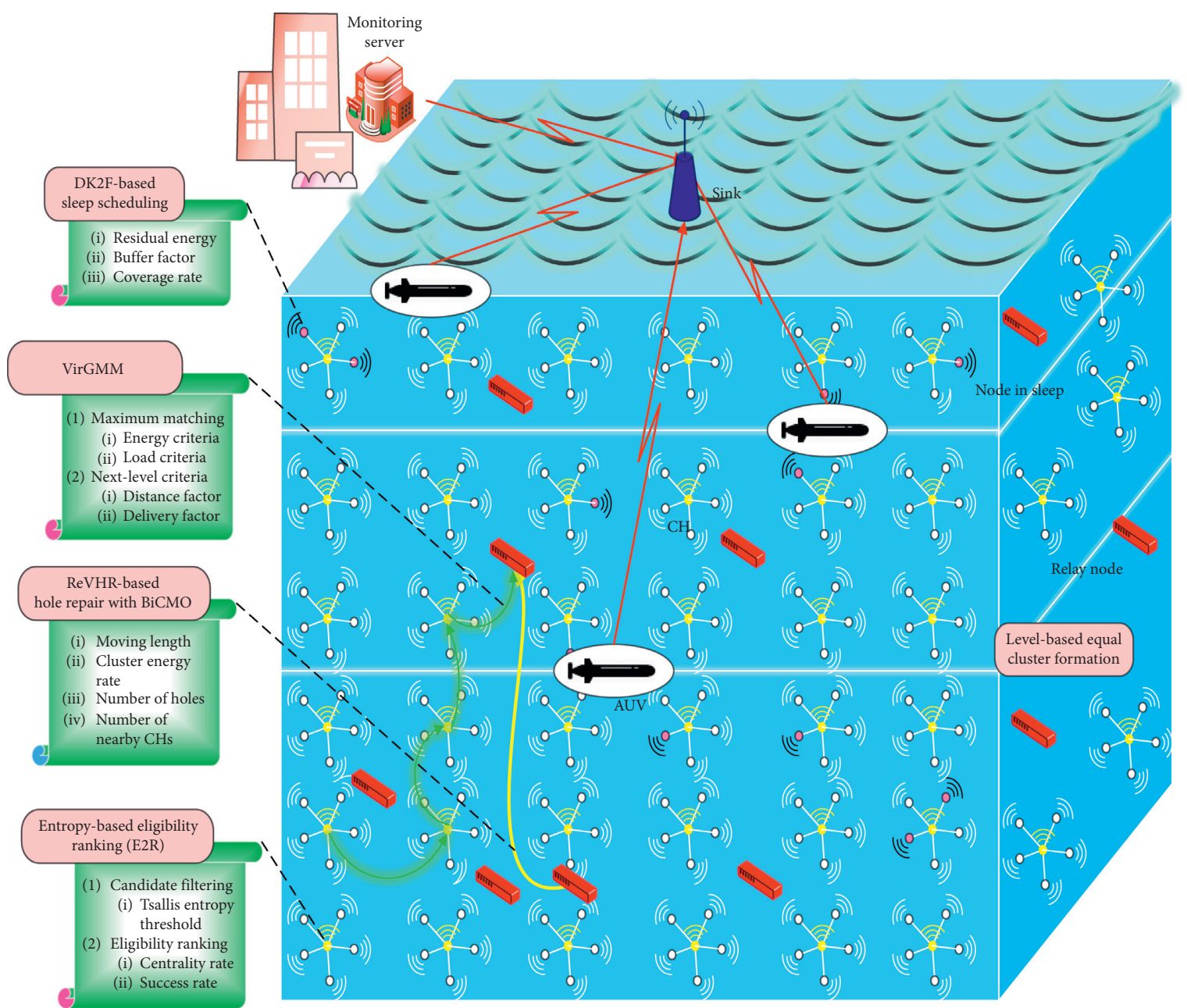

Figure 2: Network model.

Input: $\mathbf{S}_{\mathbf{i}}=\left\{\mathbf{S}_{1}, \mathbf{S}_{2}, \mathbf{S}_{3}, \ldots, \mathbf{S}_{\mathbf{N}}\right\}$,

Output: Number of Clusters (Cluster Formation and CH Selection)

Start

Step 1: $\mathbf{S}_{\mathbf{i}} \longrightarrow \mathrm{REQ}$

Step 2: if $\mathbf{S}_{\mathbf{i}} \longrightarrow \mathfrak{R}_{\xi}, \mathbf{C}_{\mathbf{f}}, \mathbf{S R}_{\mathbf{c}} / /$ higher energy, central value and success rate)

Then

$\mathrm{S}_{\mathbf{i}} \longrightarrow \mathrm{CH}_{\mathbf{i}}$

If $\left(\mathrm{CH}_{\mathbf{i}}\right.$ found $)$

$\mathrm{CM}_{\mathbf{i}} \longrightarrow \mathrm{CH}_{\mathbf{i}}$ (based on REP)

End if

End if

Step 3: if $\mathbf{C H}_{\mathbf{i}} \longrightarrow$ low $\mathfrak{R}_{\xi}, \mathbf{C}_{\mathbf{f}}, \mathbf{S R}_{\mathbf{c}}$

Then elect another $\mathrm{CH}$

$\mathbf{C H}_{\mathbf{i}} \longrightarrow$ nearer $\mathbf{C H}_{\mathbf{i}}$

End if

Step 4: $\mathbf{C H}_{\mathbf{i}} \longrightarrow \mathrm{SN}$

End 
Firstly, kernel function is initiated which is defined by

$$
\delta_{\aleph}(e)=\frac{1}{1+e^{2} / \aleph},
$$

where $e$ is the exponential term between two different variables, $\aleph$ is the dynamic kernel bandwidth, and $\delta$ is the range between 0 and $\infty$. DK2F gives the optimum solution for both linear and nonlinear cases of the model in dynamic nature. The mathematical formulation of $\mathrm{DK} 2 \mathrm{~F}$ is described as follows:

$$
\begin{aligned}
& X_{T}=f_{T} X_{T}+A_{T}, \\
& Y_{T}=h_{T} X_{T}+B_{T},
\end{aligned}
$$

where $X_{T}$ is the state vector and $Y_{T}$ is the observation measurements at time $T . f_{T}$ and $h_{T}$ represent the state transition matrix and the observation matrix, respectively. $A_{T}$ and $B_{T}$ denote the noise values in observation and Gaussian noise, respectively. In the probabilistic model, it is represented as

$$
\begin{aligned}
\rho\left(Y_{T} \mid X_{T}\right) & =N\left(Y_{T} \mid h X_{T}, a\right), \\
\rho\left(X_{T} \mid X_{T-1}\right) & =N\left(Y_{T} \mid f X_{T}, b\right) .
\end{aligned}
$$

Figure 3 illustrates the dynamic sleep scheduling model. The assumption and prediction of the underwater sensor node is illustrated as follows (Algorithm 2):

(i) Every node in underwater environment follows only three kinds of states as active, sleep, or transmit. In active state, sensors are working and listening to the surrounding events and process the computations. Besides, it can also possible to switching to the idle state.

(ii) All sensors can be possible to act as a relaying state for packet transmission to the near AUV. For that, each node maintains next hop nodes in the neighbors list.

(iii) The duration of the active status is exponentially distributed with mean $1 / S$. In the active state, the sensors will sense packets, relay packets, and process packets. When all nodes are under sleep state, the $\mathrm{CH}$ cannot aggregate or transmit any packets. In this case, entire cluster putted in OFF state. When at least one node in an active state, then $\mathrm{CH}$ turns into $\mathrm{ON}$ state again. In this case, $\mathrm{CH}$ can transmit or receive packets from cluster members. The actual and predicted result for the DK2F is indicated in Figure 4.

Input: Total Cluster Members $i$

Output: Scheduled Mode

(1) Begin

(2) Initialize $i=\left\{s_{1}, s_{2}, s_{3}, \ldots\right\} /{ }^{*}$ sensors in a cluster*/

(3) For each $i$

(4) Find $\mathfrak{R}_{\xi}, \varsigma_{f}$, and $\chi_{r}$

(5) List RE $\varsigma_{f}$, and $\chi_{r}$ for each $k$

(6) Find Dynamic Kernel Values
(7) if $\left(\mathfrak{R}_{\xi}<\mathfrak{R}_{\xi T h} \& \& \varsigma_{f}<\varsigma_{f(T h)} \& \& \chi_{r}<\varsigma_{f(T h)}\right)$

$I^{*}$ comparison with threshold*/

\{

assign sleep mode

else

assign active mode

\}

end if

(8) End for

(9) End

4.4. Virtual Graph-Based Routing. Intercluster routing is performed to improve energy efficiency and delivery rate. We present a novel Virtual Graph-enabled Maximum Matching (VirGMM) algorithm. In first step, the virtual graph is constructed for the $\mathrm{CHs}$. Then, maximum matching nodes are selected based on dual-criteria as energy criteria \{residual energy level and expected energy consumption\} and load criteria \{current load level and expected load level\}. Among maximum matching nodes, optimal forwarder is selected upon optimum criteria. The criteria are selected upon the following rules:

\{If AUV is presented in Same Level, then Choose [Delivery Rate Criteria];

\section{Else, Choose [Distance Criteria]\}}

In this way, data are transmitted to AUV through optimal forwarders. Figure 5 describes the virtual graph-based routing.

To minimize the sensor energy usage in data transmission, virtual graph with maximum matching theory is applied in which nearest next hop is selected for fast data transmission without any packet loss. Sensors in active state sense the event about the environment and then send the sensed report to the sink node through AUV and next hops.

In this algorithm, bicriteria is used such as $\mathfrak{R}_{\xi}$ and $E \Re_{\xi}$ for first criteria and $C_{L}$ and $\mathrm{EC}_{L}$ are considered as the second criteria to find the perfect match. For each node, the connectivity to become maximum match (MM) is derived from the Bayesian theory as follows:

$$
\begin{aligned}
C\left(S_{i}\left(\mathfrak{R}_{\xi}\right) \mid \mathrm{MM}\right) & =\frac{C\left(\mathrm{MM} \mid S_{i}\left(\mathfrak{R}_{\xi}\right)\right) C\left(S_{i}\left(\mathfrak{R}_{\xi}\right)\right)}{C(\mathrm{MM})} \\
C\left(S_{i}\left(E \mathfrak{R}_{\xi}\right) \mid \mathrm{MM}\right) & =\frac{C\left(\mathrm{MM} \mid S_{i}\left(E \mathfrak{R}_{\xi}\right)\right) C\left(S_{i}\left(E \mathfrak{R}_{\xi}\right)\right)}{C(\mathrm{MM})}, \\
C\left(S_{i}\left(C_{L}\right) \mid \mathrm{MM}\right) & =C\left(\mathrm{MM} \mid S_{i} C_{L}\right) C\left(\frac{S_{i}\left(C_{L}\right)}{C(\mathrm{MM})}\right. \\
C\left(S_{i}\left(\mathrm{EC}_{L}\right) \mid \mathrm{MM}\right) & =C\left(\mathrm{MM} \mid S_{i} \mathrm{EC}_{L}\right) C\left(\frac{S_{i}\left(\mathrm{EC}_{L}\right)}{C(\mathrm{MM})}\right.
\end{aligned}
$$

where equation (12) computes the probability of a node $S_{i}$ to become a next hop based on its $\mathfrak{R}_{\xi}$. Similarly, equations 


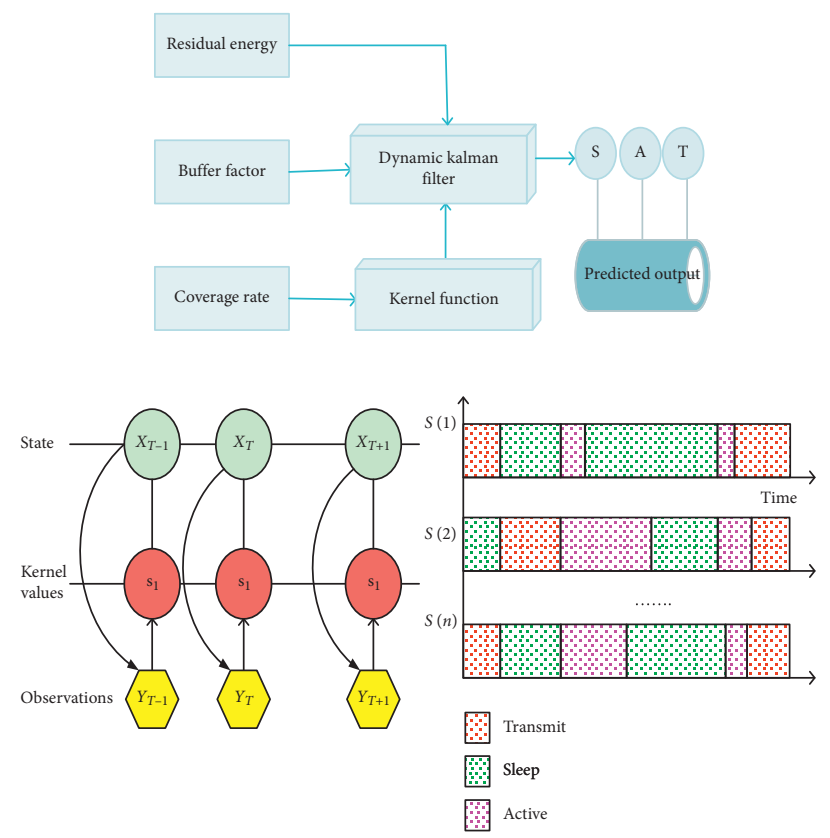

Figure 3: Dynamic sleep scheduling.

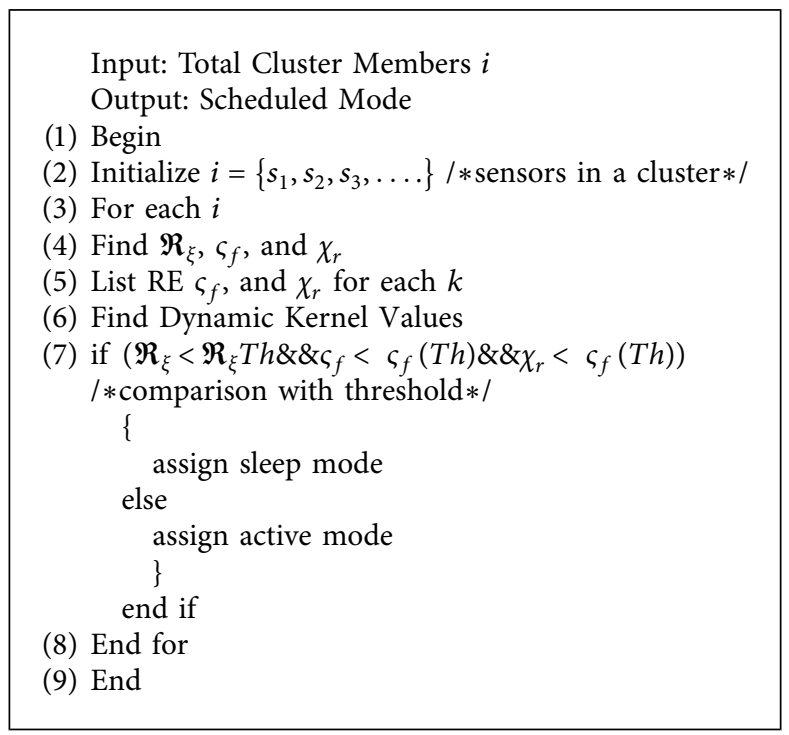

Algorithm 2: Member-balanced scheduling.

(13)-(15) compute the probability based on current load and expected load, respectively. Here, the maximum matched values are mapped between 0 and 1 range. The source $\mathrm{CH}$ found the MM for all available next hops and sorted the best set of matches. The set of possible matches by matching theory is illustrated in Table 3.

4.5. Relay-Assisted Void Hole Repair. During the route selection process when transmitting the data through different sensor nodes, a VOID hole may appear. Once the void hole is detected, then the report is generated and sent to the AUV. Mainly each $\mathrm{CH}$ in the cluster aggregates the sensor data and forwards them to the AUV through an optimal selected route, and in case a hole is detected during the routing process, the AUV then takes the optimal decision on the void hole repairing by selecting an optimal repair position by the relay nodes. In this, we introduced the novel relay-assisted void hole repair mechanism (ReVHR). On receiving void requests, the AUV selects an optimal relay node to repair the hole by replacing the failed node in the route. The relay node selection is carried based on AUV trajectory distance factor from the detected hole. Then, optimal position of the relay is determined by the bicriteria mayfly optimization (BiCMO) algorithm. The BiCMO considers the following objective functions:

$$
\mathrm{OF}=\left\{\operatorname{Min}\left[m_{l}\right] \& \& \operatorname{Min}\left[n_{(h)}\right]\right\}
$$




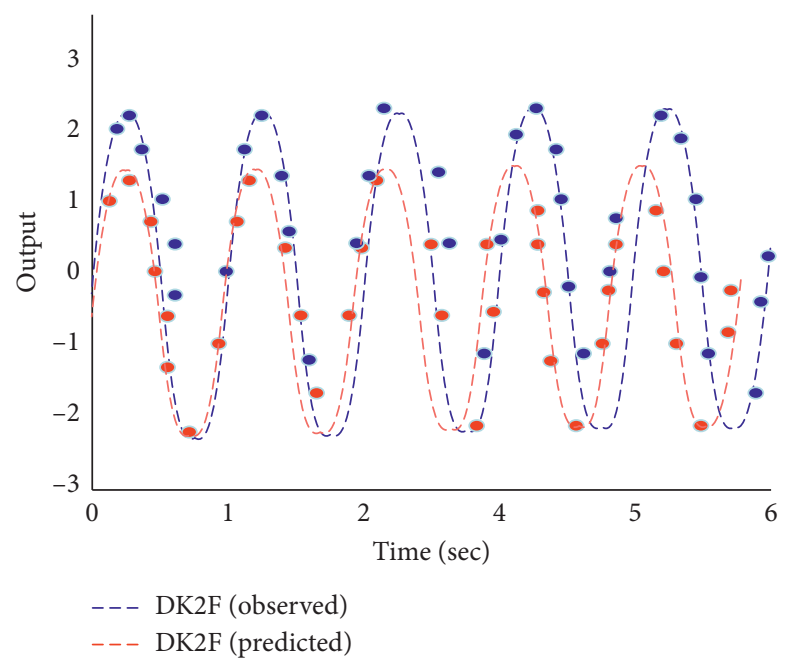

FIgURE 4: Performance plot for DK2F.

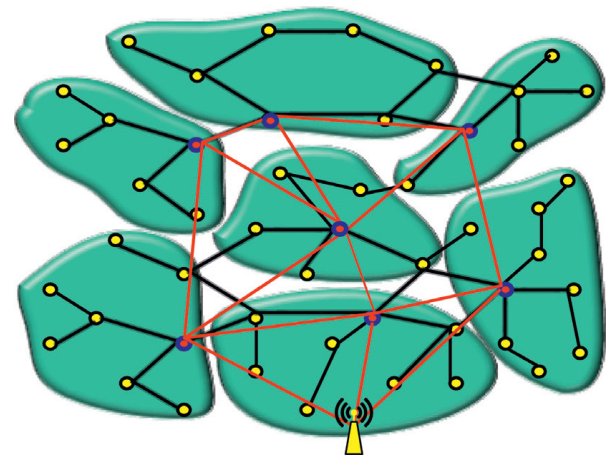

FIGURE 5: Virtual graph-based routing.

TABLE 3: Set of rules for forwarder selection.

\begin{tabular}{lcccc}
\hline $\boldsymbol{R}_{\xi}$ & $E \boldsymbol{R}_{\xi}$ & $C_{L}$ & EC $_{L}$ & Status of node \\
\hline$<0.5$ & $<0.5$ & $<0.5$ & $<0.5$ & Partially match \\
$<0.5$ & $<0.5$ & $>0.5$ & $>0.5$ & No match \\
$<0.5$ & $>0.5$ & $<0.5$ & $<0.5$ & No match \\
$<0.5$ & $>0.5$ & $>0.5$ & $>0.5$ & No match \\
$>\mathbf{0 . 5}$ & $<\mathbf{0 . 5}$ & $<\mathbf{0 . 5}$ & $<\mathbf{0 . 5}$ & Highly match \\
$>0.5$ & $<0.5$ & $>0.5$ & $>0.5$ & Partially match \\
$>0.5$ & $>0.5$ & $<0.5$ & $>0.5$ & Partially match \\
$>0.5$ & $>0.5$ & $>0.5$ & $>0.5$ & No match \\
\hline
\end{tabular}

where $m_{l}$ is the moving length and $n_{(h)}$ is the number of holes. The moving distance, number of holes, number of $\mathrm{CHs}$, and energy level of the region are considered for repositioning the void holes. Once the relay node is repositioned, the source $\mathrm{CH}$ transmits the data to AUV through the relay node. In this way, the void hole is repaired and the data are transmitted to AUV without loss.

Algorithm 3 describes the pseudocode for mayfly-based replay selection. The fitness value is computed for each node by biobjectives. The computational complexity for this algorithm is $O(N)$, where $N$ denotes the number of underwater sensors.

\section{Simulation Results}

In this section, the simulation results are presented for the proposed REVOHPR protocol is evaluated in terms of energy consumption, packet delivery ratio, and throughput for effective data transmission and hole detection and repair mechanisms. In addition, the proposed ReVHR protocol is compared to similar UWSN protocols such as ESRVR [38], 
Input: $n$ nodes' number

Output: Void Repair

Procedure:

(1) Initialize population

(2) For all sensors

(3) Estimate the fitness

(4) Find the total fitness ( ) = (1), (2), ..

(5) Compute the node probability

(6) Identification of Relay Node

If (Probability $==$ High)

Assign Node $\rightarrow$ VHR//VHR-Void Hole Repair Else

Alert broad-casted to next relay nodes

Move to next node

End if

(8) End for

(9) End

Algorithm 3: Mayfly-based relay node selection.

ACMC [40], and PSO [41]. The detailed description of the simulation environment and comparative study is specified as follows.

5.1. Simulation Setup. The simulation of the proposed vs. existing protocols for data transmission in UWSN is implemented using NS3.27. In NS3, AquaSim as shown in Figure 6 is one of the significant modules for underwater sensor environment simulation, and besides other modules, it supports to create the network model. The simulation parameters used in the proposed REVOHPR model are illustrated in Table 4. The simulation parameters are not constrained by any limit. The simulation is performed using the Ubuntu 14.04 LTS operating system with a 32 bit processor. Compared to the other simulators, NS3 is a more flexible tool to simulate clustering, sleep scheduling, intercluster routing, and void hole detection and repair mechanism. The procedure for simulation is depicted in Figure 7.

As discussed above, the simulation result is indicated in Figure 8 . The sensor data are collected by the AUV and transmitted to the onshore sink for further processing.

Figure 9 shows the flowchart of the proposed ReVOHPR protocol. This step evaluates the performance of the protocol. At the end of the simulation, graphical plots are drawn by the simulation result. Our protocol is dynamic and supported for diverse nature (applications and dynamic range of simulation).

5.2. Application Scenario: Sea Life Monitoring. The proposed protocol is tested for sea life monitoring. In this case, the sensors used are suitable for underwater animal monitoring that is deployed in the ocean and record real-time events from the environment. Two types of underwater sensors are used for sea life monitoring, i.e., physical sensors and chemical sensors. Pressure, oxygen, and temperature are the physical sensors, whereas salinity, turbidity, $\mathrm{pH}$, nitrate, chlorophyll, and dissolved oxygen are the chemical sensors.
The representation of the sea life monitoring is depicted in Figure 10. The type of sensors and their purpose is described in Table 5. The specification of each sensor is illustrated in Table 6. Ocean climate is changed over a long period which is hazardous to marine life. For example, abnormal sea temperatures affect the life of sea animals.

5.3. Comparative Study. In this section, we illustrate the performance analysis of the proposed and previous protocols in terms of various QoS and energy consumption metrics as energy consumption, delay, throughput, and packet delivery ratio (PDR). The previous protocols for data transmission and void hole repair can be follows: ESRVR [38], ACMC [40], and PSO [41].

5.3.1. Energy Consumption. Energy is a significant metric in underwater sensor communications. In the underwater sensor network, acoustic signals are transmitted in a cylindrical way. Higher energy consumption must be avoided since it represents the worst performance of the network. Packet transmission loss between two nodes is the major reason for higher energy consumption. This transmission loss $t(l)$ is computed by follows:

$$
t(l)=10 \log \frac{R_{1}}{R_{2}},
$$

where $R_{1}$ and $R_{2}$ are the source and destination nodes of the transmission. Average energy consumption is the sum of energy consumed by all nodes in the network during idle, packet transmission, reception, and sensing:

$$
E_{(i)}=\sum_{i=1}^{N} E\left(\mathrm{Tx}_{i}\right)+\sum_{i=1}^{N} E\left(\mathrm{Rx}_{i}\right)+\sum_{i=1}^{N} E\left(\mathrm{Se}_{i}\right)+\sum_{i=1}^{N} E\left(I_{i}\right) \text {, }
$$

where $N$ represents the total number of nodes, $\mathrm{Tx}_{i}$ is the sum of energy for transmission, $\mathrm{Rx}_{i}$ is the sum of energy for 


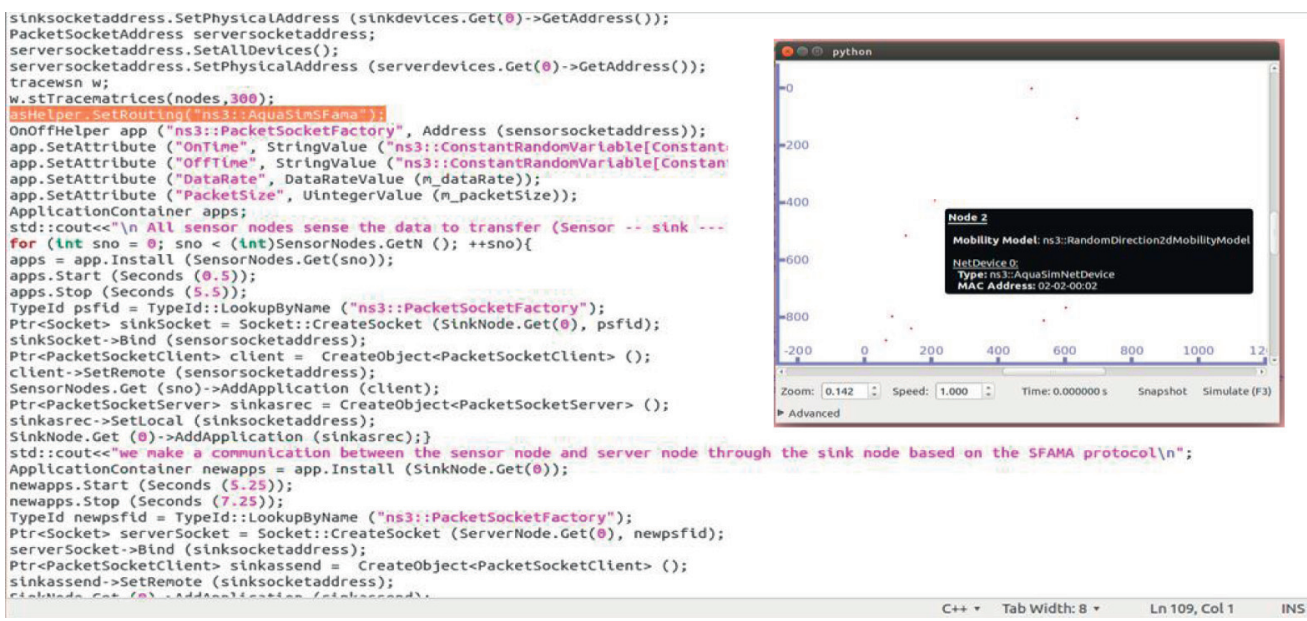

Figure 6: AquaSim module in NS3 for acoustic communication.

TABLE 4: NS3 simulation parameters.

\begin{tabular}{lcc}
\hline & & Specification \\
\hline & Simulation area & $1000 \times 1000 \times 1000$ \\
& \# of underwater & 100 \\
sensors & 10 \\
\# of relay nodes & 3 \\
& \# of AUVs & 1 \\
& Num of sink & $5-7$ \\
& Simulation time & 300 seconds \\
\hline & Modules used & AquaSim, antenna, config store, CSMA, LTE, AODV, mesh, mobility, DSR, flow monitor, \\
& Packet size & and internet \\
\hline Underwater sensor & \# of packets & $512 \mathrm{kB}$ \\
parameters & Packet time interval & $20-200$ \\
& Data rate & 100 milli seconds \\
& Initial energy per & $10-20$ Mbps \\
& sensor & $70 \mathrm{~J}$ \\
& Transmission range & $300 \mathrm{~m}$ \\
\hline
\end{tabular}

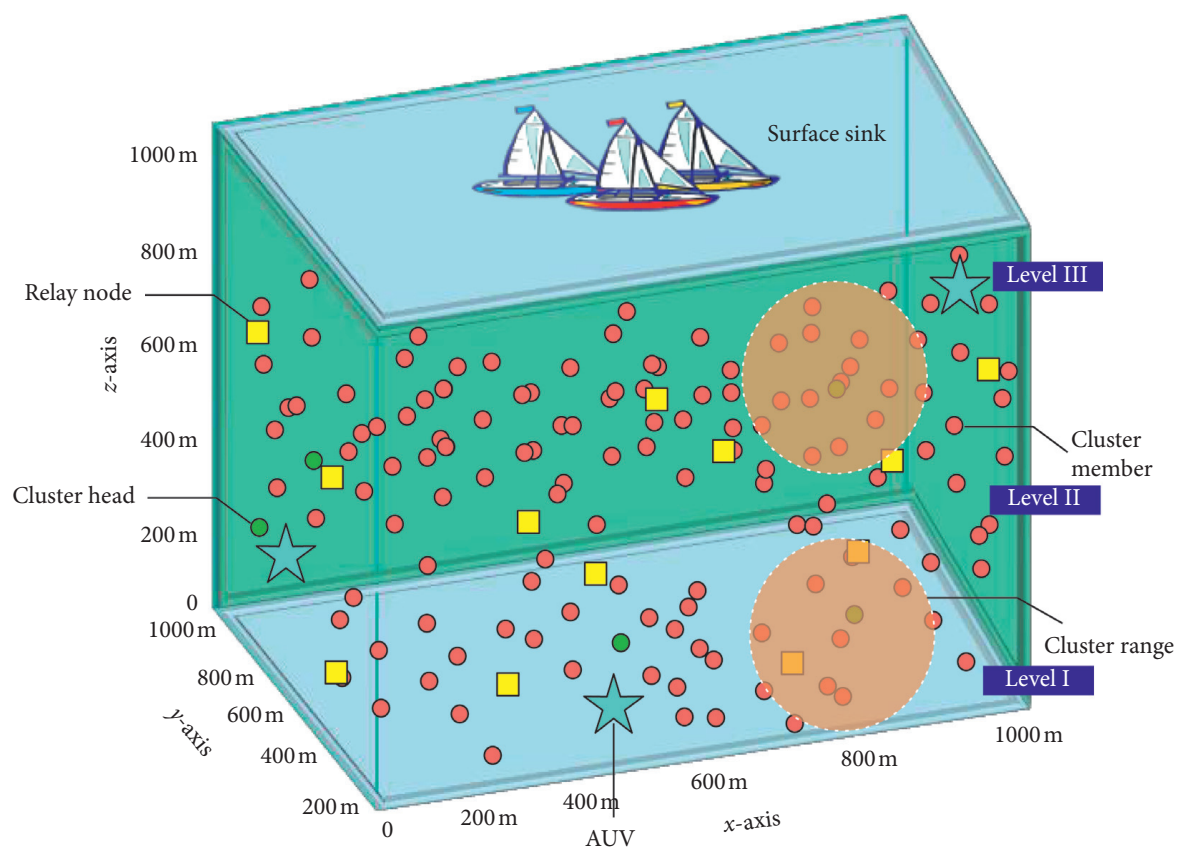

FIGURE 7: Simulation setup for REVOHPR protocol. 


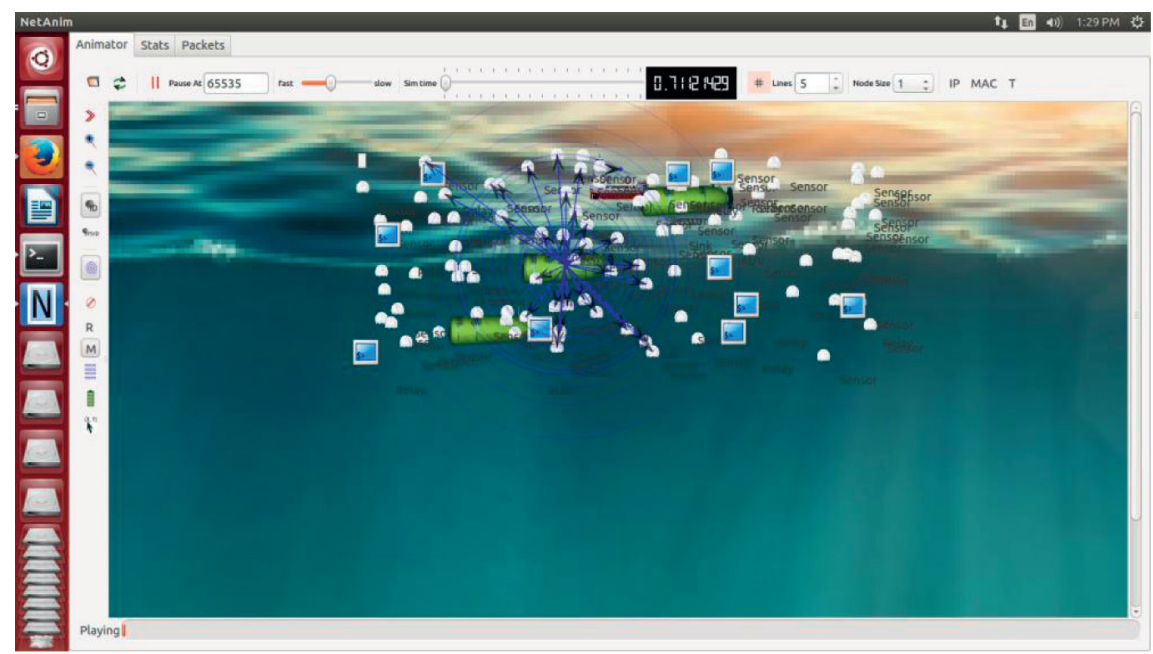

Figure 8: Simulation running in NS3.

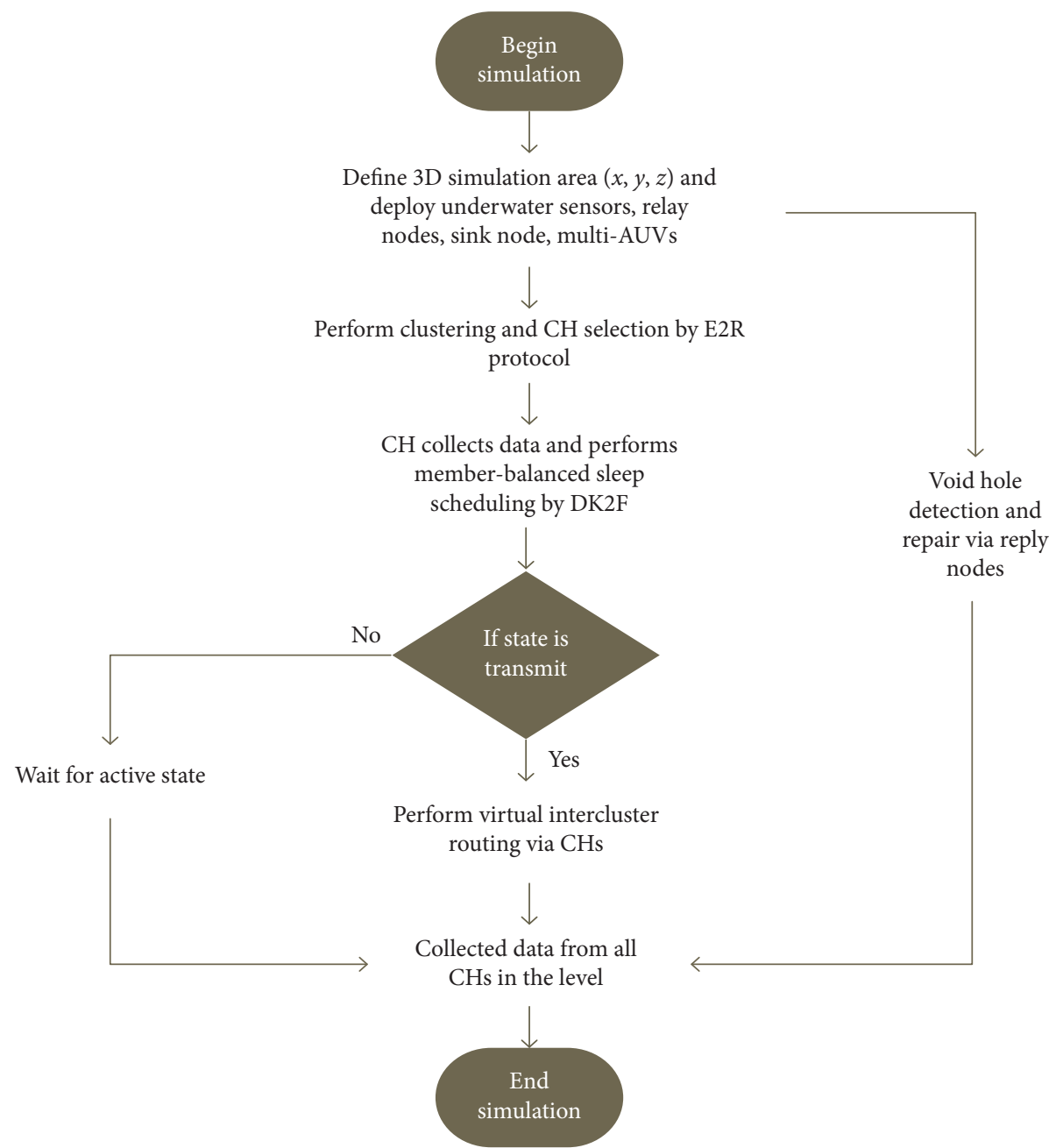

Figure 9: Flowchart for REVOHPR protocol. 


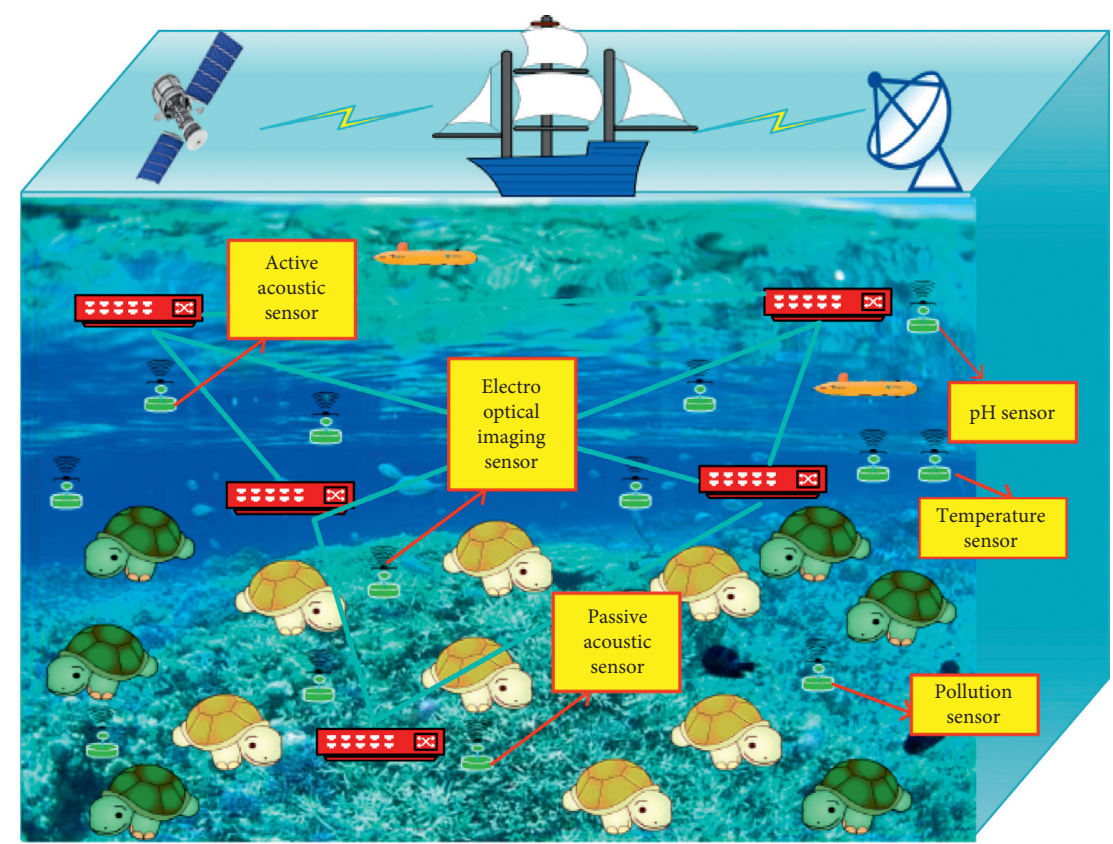

Figure 10: Sea life monitoring in UWSN.

TABle 5: Sea life sensors.

\begin{tabular}{lcc}
\hline Animal type & Animal status & Suitable sea sensors \\
\hline $\begin{array}{l}\text { Dolphins } \\
\text { Whales }\end{array}$ & Below sea surface & Active acoustic monitoring sensors \\
\hline $\begin{array}{l}\text { Seals } \\
\text { Turtles }\end{array}$ & Frequently near or at sea surface & Electro optical imaging sensors \\
\hline $\begin{array}{l}\text { Fish } \\
\text { Porpoises }\end{array}$ & Frequent distinct vocalization & Passive acoustic monitoring sensors \\
\hline
\end{tabular}

TABLE 6: Underwater sea life monitoring sensors.

\begin{tabular}{|c|c|c|c|c|c|}
\hline Sensors & Observed pattern & Range & Accuracy & Power supply & Unit \\
\hline SBE16plus V2 & Temperature & $-5-+30^{\circ} \mathrm{C}$ & $\pm 0.0055^{\circ} \mathrm{C}$ & $9-30 \mathrm{~V}$ & ${ }^{\circ} \mathrm{C}$ \\
\hline GT301 & Pressure & $0-60$ & $< \pm 0.5 \%$ of $\mathrm{FRO}$ & $24 \mathrm{~V}$ & Bar \\
\hline SBE16plus V2 & Conductivity & $0-9$ & \pm 0.0005 & $9-30 \mathrm{~V}$ & $\mathrm{~S} / \mathrm{m}$ \\
\hline OBS $-3+$ & Turbidity & Mud: $5000-10,000 \mathrm{mg} / \mathrm{L}$ & $0.5 \mathrm{NTU}$ & $15 \mathrm{~V}$ & NTU \\
\hline PS 2102 & $\mathrm{pH}$ & $0-14 \mathrm{pH}$ & \pm 0.1 & $\mathrm{~N} / \mathrm{A}$ & $\mathrm{pH}$ \\
\hline YSI 6025 & Chlorophyll & $0-400 \mu \mathrm{g} / \mathrm{L}$ & $0.1 \mu \mathrm{g} / \mathrm{L}$ & $6 \mathrm{~V}$ & $\mu \mathrm{g} / \mathrm{L}$ \\
\hline ISUS V3 & Nitrate & $0.007-28 \mathrm{mg} / \mathrm{L}$ & $\pm 0.028 \mathrm{mg} / \mathrm{L}$ & $6-18 \mathrm{~V}$ & $\mathrm{mg} / \mathrm{L}$ \\
\hline SBE 63 & Dissolved oxygen & $120 \%$ & 0.1 & $6-24 \mathrm{~V}$ & $\mathrm{mg} / \mathrm{L}$ \\
\hline
\end{tabular}

reception, $\mathrm{Se}_{i}$ is the sum of energy consumed in sensing, and $I_{i}$ is the node at idle state.

Figure 11 represents the sum of energy consumption rate of ReVOHPR against the previous protocols as ACMC, ESRVR, and PSO. Mathematical computations and number of iterations for cluster formation, $\mathrm{CH}$ selection, and routing consider more energy consumption. These processes were used to reduce the residual energy. The effective selection of $\mathrm{CH}$ and routing by the virtual graph algorithm reduce overhead in packet transmission. Furthermore, sleep scheduling idea is used which saves energy and improves the network lifetime. The graphical plots show that the amount of energy consumption increases with respect to the number of nodes. However, network density is the primary factor that affects the network performance in terms of energy consumption and QoS. Figure 11 shows the performance of average energy consumption with respect to the number of packets per second. Data transmission is performed in a multihop fashion. When the sensors in the network communicate to the surface sink directly by single-hop fashion, 


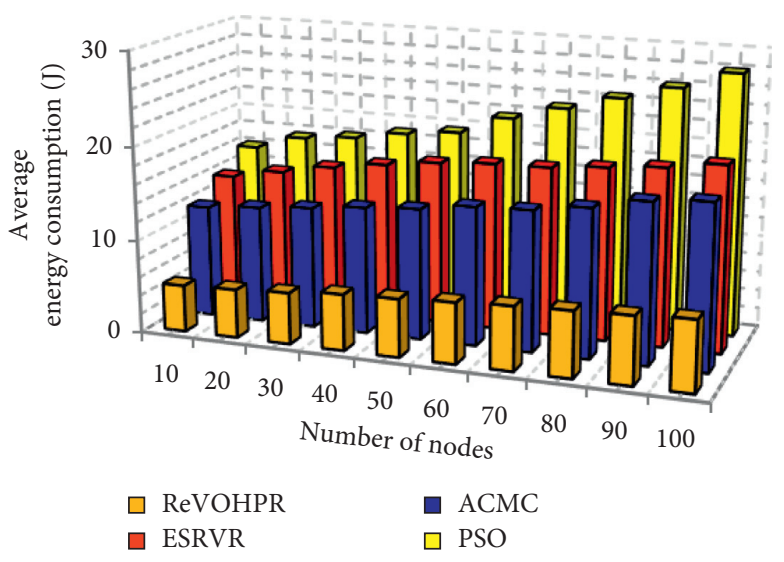

(a)

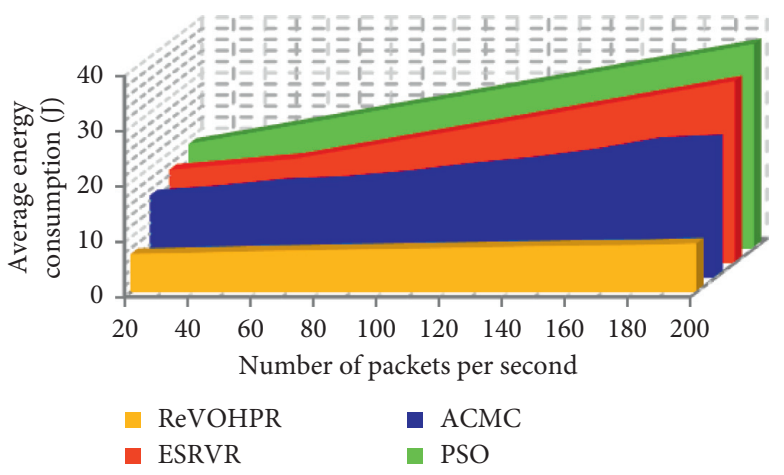

(b)

FIGURE 11: Impact of energy consumption.

then the energy consumption rate is higher. In addition, single-hop and multihop network performance is not similar in energy consumption rate. Distance between the source to the sink is higher or lesser in single hop whereas multihop routing often has a smaller number of relay nodes. In PSO and ESRVR, packets are transmitted in single hop to the surface sink. Compared to ACMC, ESRVR, and PSO, the proposed ReVOHPR consumes minimum energy, i.e., $37 \%$, $42 \%$, and $67 \%$, respectively.

5.3.2. Delay. It is the amount of time required for data transmission from source (underwater sensor) $P_{(S)}$ to the destination node (surface) $P_{(D)}$. In other words, delay is computed from the generation to the destination reception. The successful packets are counted up in delay computation. This is computed as follows:

$$
D=P_{(S)}-P_{(D)} .
$$

Figure 12 shows the performance of delay with respect to the number of nodes. The proposed ReVOHPR forwards packets by $\mathrm{CH}$, and aggregated packets forward to next hop using the multihop routing algorithm. Ocean depth is the major element to consider in both clustering and routing. When the depth of ocean is higher, then the packet collection time is higher. The delay is higher in PSO when it processes with packets without processing the void hole detection and prevention. The void management in the proposed ReVOHPR protocol improves the network data transmission and improves the node's presence. Thus, the delay in packet transmission is eliminated. The PSO-based algorithm is not sufficient for data transmission. Since, it has a low convergence rate in the iterations.

The total delay required for data transmission from source to the destination with respect to the number of packets is very higher for previous protocols. It is illustrated in Figure 12. It is computed by several factors as propagation delay, transmission delay, number of hop counts, and distance between two nodes. ReVOHPR utilizes the relay-based void hole prevention and repair. This efficiently helps to identify the presence of void and handles it precisely. The increased frequency of void occurrence increases delay when the number of nodes increases for the lengthy route. The computational time required in high traffic congestion is exponential, and it does not suit for event-based data transmission.

5.3.3. Throughput. It is the positive metric that defines the amount of packet transmitted in a time. When measuring the maximum throughput in packet transmission, then the communication link or network access is reliable. It is computed as follows:

$$
\text { Throughput }\left(\frac{\text { bits }}{\mathrm{sec}}\right)=\frac{\operatorname{Sum}(N(\mathrm{Sp}) * \text { APS })}{T(t)},
$$

where $N(\mathrm{Sp})$ is the number of successful packets, APS is the average packet size, and $T(t)$ is the total time spend for packet transmission. The simulation results in Figure 13 show that the performance of the proposed ReVOHPR is higher than the previous protocols. It is analyzed by both number of nodes and the number of packets per second.

Due to the less traffic congestion and immediate route identification from $\mathrm{CH}$ to the AUV, throughput for transmitted packets is high. Unfortunately, existing works have obtained high communication overhead and high traffic congestion. When network size expands, then the performance of throughput is low. Hence, ACMC is not able to handle the high volume of traffic. ESRVR is suited only when the network has limited number of nodes and processing with limited number of communications. In addition, it uses two-hop information for routing packets. This is insufficient in achieving higher throughput. A large number of void holes decrease network performance. In this case, frequent void hole mitigation is important that degrades network throughput.

Figure 13 shows the performance throughput with respect to the number of packets processed per second. Relaybased void hole detection and mitigation addresses and avoids the multivoid hole prediction. This problem improves energy efficiency and QoS-based metrics such as throughput, 


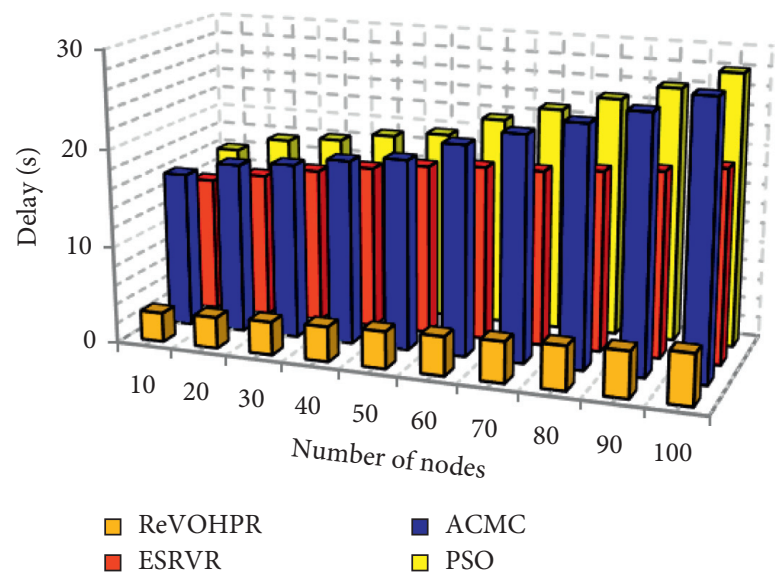

(a)

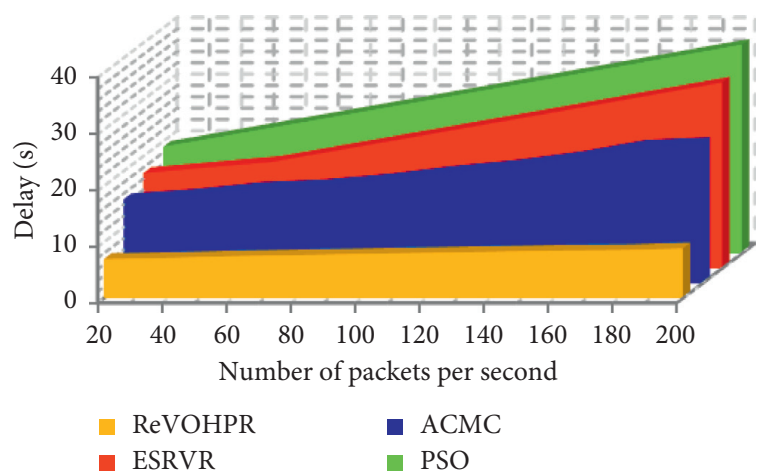

(b)

Figure 12: Impact of delay.

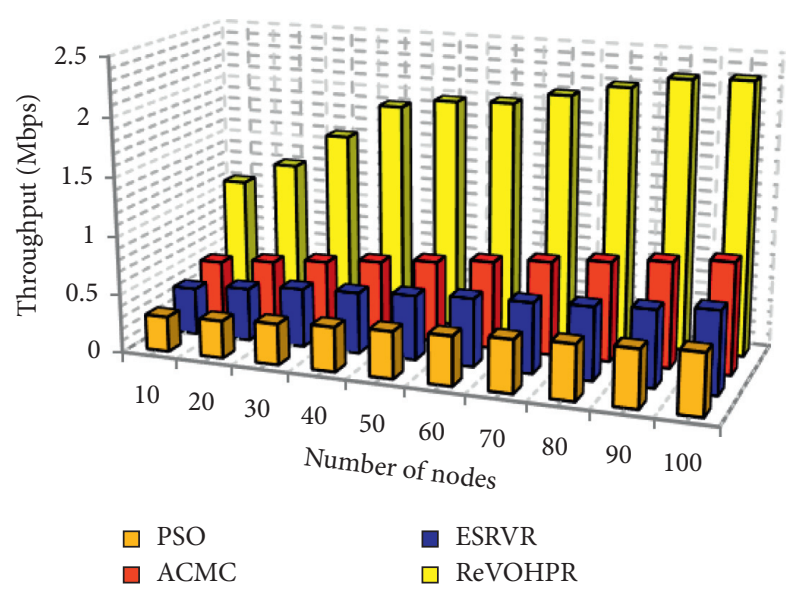

(a)

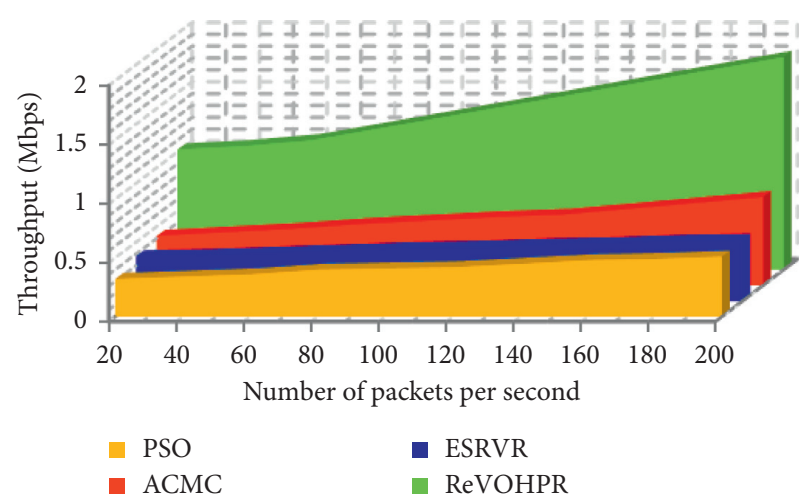

(b)

FIgURE 13: Impact of throughput.

delay, and PDR. In ACMC, clusters are formed using the $\mathrm{K}$-means algorithm in which centroid selection must be optimum and cluster size must be known. Data transfer time and mode are frequently changed and adapted in the proposed protocol. Hence, we obtained higher throughput than the previous protocols. We have used the end-to end approach to improve the network throughput for a longer period.

5.3.4. $P D R$. PDR is the packet delivery rate metric analyzed for every node in the network in data transmission time. It is defined as the sum of packets successfully received at the destination node from the source node. It is computed as follows:

$$
\mathrm{PDR}=\frac{N(P(R))}{N(P(G))},
$$

where $N(P(R))$ is the number of packets received at the source node and $N(P(G))$ is the number of packets generated at the source node.
Figure 14 shows the simulation results for the PDR with respect to the number of nodes and number of packets processed per second. The plot of PDR in ReVOHPR increases when the network density increases. Optimum relay selection for routing packets from source $\mathrm{CH}$ to the destination $\mathrm{CH}$ improves the PDR, and also a number of void holes are detected and mitigated in the proposed protocol. The existing protocol, i.e., ACMC, uses simple void handling procedure that failed since single metric is considered for void hole detection. On behalf of void hole mitigation, packets transmitted to the next hop are guaranteed, and also it ensures the packet delivery. When void hole is determined, then relay node is near to use as a replacing part, and in contrast, a previous protocol such as PSO and ESRVR does not fit for robust data transmission. In PSO, two-level $\mathrm{CHs}$ are elected which increases overhead in data transmission. Furthermore, in PSO, void hole prediction consumes more processing and hence packet losses are very high whereas ESRVR uses two hops for data transmission. The selection for two hops is not reliable in this work. Hence, PDR is very lower. 


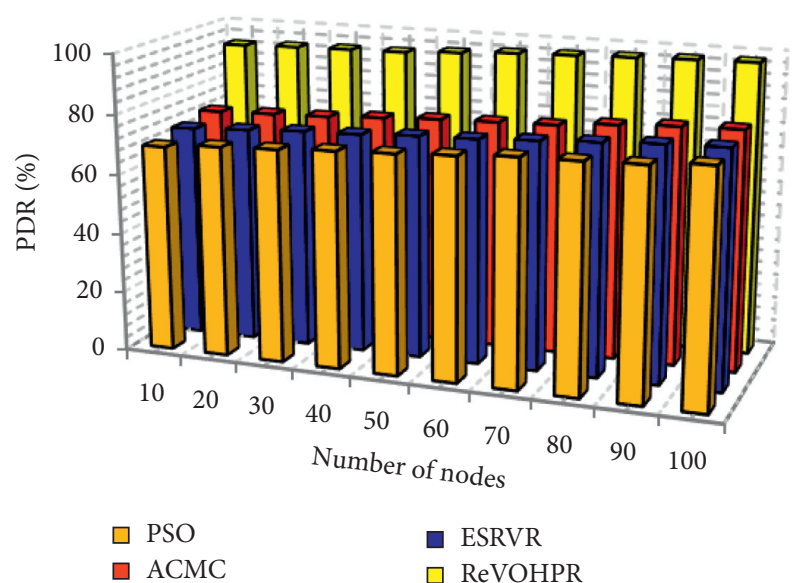

(a)

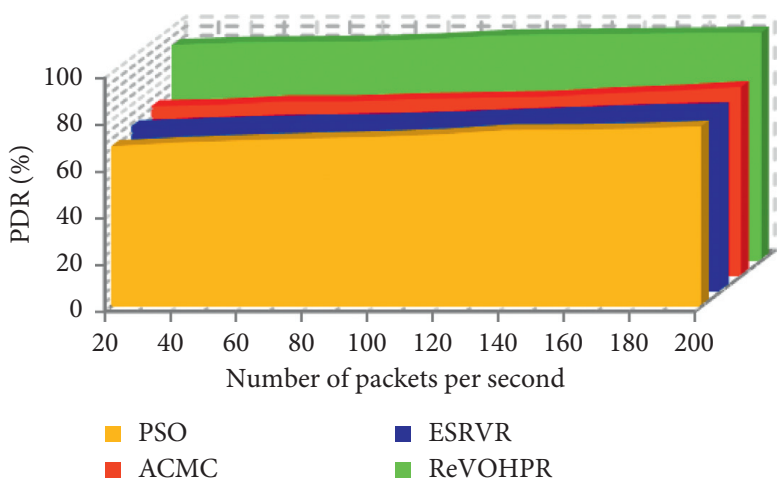

(b)

FIgURE 14: Impact of PDR.

5.4. Results and Discussion. Based on the simulation results of the proposed REVOHPR protocol with the previous protocols, it is concluded that the REVOHPR offers various benefits. Due to the development of a new protocol for data transmission and void hole mitigation in UWSN, the number of active nodes rate increases, where the dead node count is reduced. The minimization of the overall energy consumption and the improvement of the overall QoS in the proposed REVOHPR is achieved due to the following set of processes implemented.

(i) Cluster formation and optimum $\mathrm{CH}$ selection by E2R protocol that improves these processes and avoids frequent cluster formation by considering the centrality factor and success rate of each node. By this method $37 \%$ of energy consumption is reduced.

(ii) Void hole repair algorithm addresses the packet dropping issues and also eliminates the packet retransmission.

(iii) Virtual graph construction process reduces the complexity, which increases the lifetime of network than the previous protocols.

\section{Conclusion}

In this paper, the void hole problem is addressed for energy consumption reduction. For this purpose, ReVOHPR protocol is proposed which deals with the four processes, the level-based clustering in which E2R protocol is presented for stable $\mathrm{CH}$ selection. Then, dynamic sleep scheduling mechanism is considering to improve the lifetime of a network which is dynamic by implementing the DK2F algorithm. The virtual graph-based routing algorithm is presented for data transmission in which virtual route is established between the source $\mathrm{CH}$ and the destination. To avoid data transmission delay, multiple AUVs deployed to gather data packets. Finally, relay-assisted void hole detection and repair is presented which eliminates the multiple void hole problems for a longer period. Our simulation results show that the proposed ReVOHPR protocol exceeds the performance than baseline protocols as ESRVR, ACMC, and PSO in terms of energy consumption, packet delivery ratio, throughput, and delay.

In the future, we planned to focus on the security aspect of data transmission to avoid threats in UWSN. In this case, various attacks in UWSN are detected and mitigated to further reduce the energy consumption and improve the QoS [42-44].

\section{Data Availability}

The data used to support the findings of this study are available from the corresponding author upon request.

\section{Conflicts of Interest}

The authors declare that they have no conflicts of interest.

\section{Acknowledgments}

This work was supported by the RUDN University Strategic Academic Leadership Program and by the Prince Sattam Bin Abdulaziz University, Saudi Arabia, via the Deanship for Scientific Research funding for the Advanced Computational Intelligence and Intelligent Systems Engineering (ACIISE) Research Group, project number 2020/01/12173.

\section{References}

[1] G. Yang, L. Dai, G. Si, S. Wang, and S. Wang, Challenges and Security Issues in Underwater Wireless Sensor Networks, IIKI, Beijing, China, 2018.

[2] N. Kanthimathi and Dejey, "Void handling using geo-opportunistic routing in underwater wireless sensor networks," Computers \& Electrical Engineering, vol. 64, pp. 365-379, 2017.

[3] R. W. L. Coutinho, A. Boukerche, L. F. M. Vieira, and A. A. F. Loureiro, "Performance modeling and analysis of void-handling methodologies in underwater wireless sensor networks," Computer Networks, vol. 126, pp. 1-14, 2017. 
[4] M. Ahmed, M. Salleh, and M. I. Channa, "Routing protocols based on protocol operations for underwater wireless sensor network: a survey," Egyptian Informatics Journal, vol. 19, no. 1, pp. 57-62, 2018.

[5] S. M. Ghoreyshi, A. Shahrabi, and T. Boutaleb, "A clusterbased mobile data-gathering scheme for underwater sensor networks," in Proceedings of the 2018 International Symposium on Networks, Computers and Communications (ISNCC), June 2018.

[6] J. Ma, S. Shi, X. Gu, and F. Wang, "Heuristic mobile data gathering for wireless sensor networks via trajectory control," International Journal of Distributed Sensor Networks, vol. 16, pp. 1-12, 2020.

[7] Y. Liu, J. Peng, J. Kang, A. M. Iliyasu, D. Niyato, and A. A. A. El-Latif, "A secure federated learning framework for 5G networks," IEEE Wireless Communications, vol. 27, no. 4, pp. 24-31, 2020.

[8] V. Artem, M. Al-Sveiti, I. A. Elgendy, A. S. Kovtunenko, and A. Muthanna, "Detection and recognition of moving biological objects for autonomous vehicles using intelligent edge computing/LoRaWAN mesh system, lecture notes in computer science," Internet of Things, Smart Spaces, and Next Generation Networks and Systems, Springer, Cham, Switzerland, pp. 3-15, 2020.

[9] R. Shakila and B. Paramasivan, "Performance analysis of submarine detection in underwater wireless sensor networks for naval application," Microprocessors and Microsystems, vol. 13, Article ID 103293, 2020.

[10] D. Wang, J. Liu, and D. Yao, “An energy-efficient distributed adaptive cooperative routing based on reinforcement learning in wireless multimedia sensor networks," Computer Networks, vol. 178, no. 4, Article ID 107313, 2020.

[11] M. Jouhari, K. Ibrahimi, H. Tembine, and J. Ben-Othman, "Underwater wireless sensor networks: a survey on enabling technologies, localization protocols, and internet of underwater things," IEEE Access, vol. 7, pp. 96879-96899, 2019.

[12] M. Khayyat, A. Alshahrani, S. Alharbi, I. Elgendy, A. Paramonov, and A. Koucheryavy, "Multilevel serviceprovisioning-based autonomous vehicle applications," Sustainability, vol. 12, no. 6, p. 2497, 2020.

[13] S. Basagni, V. D. Valerio, P. Gjanci, and C. Petrioli, "MARLIN-Q: multi-modal communications for reliable and low-latency underwater data delivery," Ad Hoc Networks, vol. 82, pp. 134-145, 2018.

[14] N. Ismat, R. Qureshi, R. N. Enam, S. Noor, and M. Tahir, "Cluster estimation in terrestrial and underwater sensor networks," Wireless Personal Communications, vol. 116, no. 2, pp. 1443-1462, 2020.

[15] M. Zhang and W. Cai, "Energy-efficient depth based probabilistic routing within 2-hop neighborhood for underwater sensor networks," IEEE Sensors Letters, vol. 4, no. 6, pp. 1-4, 2020.

[16] M. Ahmed, M. Salleh, and M. I. Channa, "Routing protocols for underwater wireless sensor networks based on data forwarding: a review," Telecommunication Systems, vol. 65, no. 1, pp. 139-153, 2016.

[17] Z. Wang, G. Han, H. Qin, S. Zhang, and Y. Sui, "An energyaware and void-avoidable routing protocol for underwater sensor networks," IEEE Access, vol. 6, pp. 7792-7801, 2018.

[18] M. Ahmed, M. Salleh, and M. I. Channa, "CBE2R: clusteredbased energy efficient routing protocol for underwater wireless sensor network," International Journal of Electronics, vol. 105, no. 11, pp. 1916-1930, 2018.
[19] M. Chen and D. Zhu, "Data collection from underwater acoustic sensor networks based on optimization algorithms," Computing, vol. 102, no. 1, pp. 83-104, 2019.

[20] A. Jamshidi, "Efficient cooperative ARQ protocols based on relay selection in underwater acoustic communication sensor networks," Wireless Networks, vol. 25, no. 8, pp. 4815-4827, 2018.

[21] N. Javaid, F. Ahmed, Z. Wadud, N. Alrajeh, M. Alabed, and M. Ilahi, "Two hop adaptive vector based quality forwarding for void hole avoidance in underwater WSNs," Sensors, vol. 17, 2017.

[22] N. Javaid, O. Karim, A. Sher, M. Imran, A. Yasar, and M. Guizani, "Q-Learning for energy balancing and avoiding the void hole routing protocol in underwater sensor networks," in Proceedings of the 2018 14th International Wireless Communications \& Mobile Computing Conference (IWCMC), pp. 702-706, Limassol, Cyprus, June 2018.

[23] G. Latif, N. Javaid, A. Sher, M. Khan, T. Hameed, and W. Abbas, "An efficient routing algorithm for void hole avoidance in underwater wireless sensor networks," in Proceedings of the 2018 IEEE 32nd International Conference on Advanced Information Networking and Applications (AINA), pp. 305-310, Krakow, Poland, May 2018.

[24] A. Sher, A. Khan, N. Javaid, S. H. Ahmed, M. Y. Aalsalem, and W. Khan, "Void hole avoidance for reliable data delivery in IoT enabled underwater wireless sensor networks," Sensors, vol. 18, 2018.

[25] A. Signori, F. Campagnaro, F. Steinmetz, B.-C. Renner, and M. Zorzi, "Data gathering from a multimodal dense underwater acoustic sensor network deployed in shallow fresh water scenarios," Journal of Sensor and Actuator Networks, vol. 8, no. 4, p. 55, 2019.

[26] F. Banaeizadeh and A. Toroghi Haghighat, "An energy-efficient data gathering scheme in underwater wireless sensor networks using a mobile sink," International Journal of Information Technology, vol. 12, no. 2, pp. 513-522, 2020.

[27] S. Kumari, P. K. Mishra, and V. Anand, "Fault resilient routing based on moth flame optimization scheme for underwater wireless sensor networks," Wireless Networks, vol. 26, no. 2, pp. 1417-1431, 2020.

[28] S. M. Ghoreyshi, A. Shahrabi, T. Boutaleb, and M. Khalily, "Mobile data gathering with hop-constrained clustering in underwater sensor networks," IEEE Access, vol. 7, pp. 21118-21132, 2019.

[29] M. T. R. Khan, S. H. Ahmed, and D. Kim, "AUV-aided energy-efficient clustering in the internet of underwater things," IEEE Transactions on Green Communications and Networking, vol. 3, no. 4, pp. 1132-1141, 2019.

[30] W. Zhang, J. Wang, G. Han, X. Zhang, and Y. Feng, "A cluster sleep-wake scheduling algorithm based on 3D topology control in underwater sensor networks," Sensors, vol. 19, 2019.

[31] T. Islam and S.-H. Park, "A two-stage routing protocol for partitioned underwater wireless sensor networks," Symmetry, vol. 12 , no. 5 , p. $783,2020$.

[32] Z. Wadud, M. Ismail, A. B. Qazi et al., "An energy balanced efficient and reliable routing protocol for underwater wireless sensor networks," IEEE Access, vol. 7, pp. 175980-175999, 2019.

[33] Q. Guan, F. Ji, Y. Liu, H. Yu, and W. Chen, "Distance-vector-based opportunistic routing for underwater acoustic sensor networks," IEEE Internet of Things Journal, vol. 6, no. 2, pp. 3831-3839, 2019.

[34] M. Akbar, N. Javaid, A. Khan, M. Imran, M. Shoaib, and A. Vasilakos, "Efficient data gathering in $3 \mathrm{D}$ linear 
underwater wireless sensor networks using sink mobility," Sensors, vol. 16, no. 3, p. 404, 2016.

[35] M. Faheem, M. A. Ngadi, and V. C. Gungor, "Energy efficient multi-objective evolutionary routing scheme for reliable data gathering in internet of underwater acoustic sensor networks," Ad Hoc Networks, vol. 93, Article ID 101912, 2019.

[36] V. Krishnaswamy and S. Manvi, "Fuzzy and PSO based clustering scheme in underwater acoustic sensor networks using energy and distance parameters," Wireless Personal Communications, vol. 108, no. 3, pp. 1529-1546, 2019.

[37] M. Awais, I. Ali, T. A. Alghamdi et al., "Towards void hole alleviation: enhanced GEographic and opportunistic routing protocols in harsh underwater WSNs," IEEE Access, vol. 8, pp. 96592-96605, 2020.

[38] A. Khan, K. Aurangzeb, E.-U.-H. Qazi, and A. Ur Rahman, "Energy-aware scalable reliable and void-hole mitigation routing for sparsely deployed underwater acoustic networks," Applied Sciences, vol. 10, no. 1, p. 177, 2019.

[39] Q. Wang, J. Li, Q. Qi, P. Zhou, and D. O. Wu, "A game theoretic routing protocol for 3D underwater acoustic sensor networks," IEEE Internet of Things Journal, vol. 7, no. 10, pp. 9846-9857, 2020.

[40] M. Huang, K. Zhang, Z. Zeng, T. Wang, and Y. Liu, “An AUV-assisted data gathering scheme based on clustering and matrix completion for Smart ocean," IEEE Internet of Things Journal, vol. 7, no. 10, pp. 9904-9918, 2020.

[41] Z. Jin, Q. Zhao, and Y. Luo, "Routing void prediction and repairing in AUV-assisted underwater acoustic sensor networks," IEEE Access, vol. 8, pp. 54200-54212, 2020.

[42] K. Abbas, L. A. A. Tawalbeh, A. Rafiq et al., "Convergence of blockchain and IoT for secure transportation systems in smart cities," Security and Communication Networks, vol. 2021, Article ID 5597679, 13 pages, 2021.

[43] G. N. Nguyen, N. H. L. Viet, M. Elhoseny, K. Shankar, B. B. Gupta, and A. A. A. El-Latif, "Secure blockchain enabled cyber-physical systems in healthcare using deep belief network with ResNet model," Journal of Parallel and Distributed Computing, vol. 153, pp. 150-160, 2021.

[44] W. Z. Zhang, I. A. Elgendy, M. Hammad et al., "Secure and optimized load balancing for multi-tier IoT and edge-cloud computing systems," IEEE Internet of Things Journal, vol. 8, no. 10, pp. 8119-8132, 2020. 